\title{
A spectral theorem for the semigroup generated by a class of infinitely many master equations
}

\author{
Sabine Bögli* and Pierre-A. Vuillermot**,*** \\ Department of Mathematical Sciences, Durham University, \\ Durham DH1 3LE, United Kingdom* \\ UMR-CNRS 7502, Inst. Élie Cartan de Lorraine, Nancy, France** \\ Grupo de Física Matemática, GFMUL, Faculdade de Ciências, \\ Universidade de Lisboa, 1749-016 Lisboa, Portugal ${ }^{* * *}$
}

\begin{abstract}
In this article we investigate the spectral properties of the infinitesimal generator of an infinite system of master equations arising in the analysis of the approach to equilibrium in statistical mechanics. The system under consideration thus consists of infinitely many first-order differential equations governing the time evolution of probabilities susceptible of describing jumps between the eigenstates of a differential operator with a discrete point spectrum. The transition rates between eigenstates are chosen in such a way that the so-called detailed balance conditions are satisfied, so that for a large class of initial conditions the given system possesses a global solution which converges exponentially rapidly toward a time independent probability of Gibbs type. A particular feature and a challenge of the problem under consideration is that in the infinite-dimensional functional space where the initial-value problem is well posed, the infinitesimal generator is realized as a non normal and non dissipative compact operator, whose spectrum therefore does not exhibit a spectral gap around the zero eigenvalue.
\end{abstract}

\section{Introduction and outline}

In the simplest setting a system of master equations refers to a set of first-order linear ordinary differential equations which describe the time evolution of probabilities susceptible of describing transitions between the eigenstates of a given differential operator with a discrete point spectrum. As such master equations play an important role in the analysis of certain chemical reaction processes, radioactive decay processes and the propagation of epidemics, to name only a few. More generally, they also allow one to establish an important formal link between the laws that govern the microscopic and reversible motion of particles of physical or chemical systems and the macroscopic irreversible laws of thermodynamics, thereby providing some understanding of the approach to equilibrium 
in statistical mechanics (we refer the reader for instance to [7] and to Chapter V in 15. for a history and many examples, and to [9], 13], 14] with their plethora of references for recent advances in the subject of Stochastic Thermodynamics). It is precisely this last aspect that we shall be concerned with in this article, and accordingly we shall organize the remaining part of this article in the following way: In Section 2 we start out with a sequence of real numbers $\left(\lambda_{\mathrm{m}}\right)_{\mathrm{m} \in \mathbb{N}^{+}}$chosen in such a way that the so-called partition function satisfies

$$
Z_{\beta}:=\sum_{\mathrm{m}=1}^{+\infty} \exp \left[-\beta \lambda_{\mathrm{m}}\right]<+\infty
$$

for all $\beta>0$, which implies in particular that $\lambda_{\mathrm{m}} \rightarrow+\infty$ as $\mathrm{m} \rightarrow+\infty$. We then define the Gibbs probability vectors $\mathbf{p}_{\beta}$, Gibbs by their components

$$
p_{\beta, \mathrm{m}, \text { Gibbs }}:=Z_{\beta}^{-1} \exp \left[-\beta \lambda_{\mathrm{m}}\right]
$$

for every $\mathrm{m} \in \mathbb{N}^{+}$, and with the sequence $\left(\lambda_{\mathrm{m}}\right)_{\mathrm{m} \in \mathbb{N}^{+}}$also consider the class of initial-value problems for master equations of the form

$$
\begin{aligned}
\frac{d p_{\mathrm{m}}(\tau)}{d \tau} & =\sum_{\mathrm{n}=1}^{+\infty}\left(r_{\mathrm{m}, \mathrm{n}} p_{\mathrm{n}}(\tau)-r_{\mathrm{n}, \mathrm{m}} p_{\mathrm{m}}(\tau)\right), \quad \tau \in[0,+\infty), \\
p_{\mathrm{m}}(0) & =p_{\mathrm{m}}^{*}
\end{aligned}
$$

where $\left(p_{\mathrm{m}}^{*}\right)_{\mathrm{m} \in \mathbb{N}^{+}}$stands for any sequence of initial-data satisfying

$$
p_{\mathrm{m}}^{*} \geq 0, \quad \sum_{\mathrm{m}=1}^{+\infty} p_{\mathrm{m}}^{*}=1
$$

In the preceding expression the time-independent transition rates $r_{\mathrm{m}, \mathrm{n}}>0$ depend on $\lambda_{\mathrm{m}}$ and $\lambda_{\mathrm{n}}$ in a very specific way which we describe below, a choice that allows us to prove the existence of a global solution to (3) and provide a detailed investigation of the long-time behavior of each $p_{\mathrm{m}}(\tau)$ as $\tau \rightarrow+\infty$. More specifically, by interpreting (3) as a dynamical system defined in the usual Hilbert space $l_{\mathbb{C}}^{2}$ consisting of all square summable complex sequences, we provide a complete spectral analysis of the underlying infinitesimal generator $A$, which is realized there as a non normal and non dissipative compact operator. In order to get compactness we first show that $A$ is trace-class, then prove that $\nu_{1}=0$ is a simple eigenvalue of $A$ whose eigenspace is generated by $\mathrm{p}_{\beta, \text { Gibbs }}$, proceed by proving that all the remaining eigenvalues are simple, negative, and that the corresponding eigenvectors together with $\mathbf{p}_{\beta}$, Gibbs constitute a complete system in $l_{\mathbb{C}}^{2}$ in the sense of Chapter $\mathrm{V}$ in [5]. This is done very indirectly as we have to show as a preliminary step that $A$ does not possess any root vectors of height larger than one. Then, by imposing additional constraints on the sequence $\left(\lambda_{\mathrm{m}}\right)_{\mathrm{m} \in \mathbb{N}^{+}}$and by proving some delicate estimates related to the localization properties of the eigenvalues of $A$, we finally prove that the complete system in question is actually a basis of $l_{\mathbb{C}}^{2}$. This eventually leads to the spectral 
decomposition of the semigroup $\exp [\tau A]_{\tau \in[0,+\infty}$ generated by $A$, and thereby to the exponentially rapid convergence

$$
p_{\mathrm{m}}(\tau) \rightarrow \gamma p_{\beta, \mathrm{m}, \text { Gibbs }}
$$

for each $\mathrm{m}$ as $\tau \rightarrow+\infty$ for some $\gamma \in \mathbb{C}$ for a large class of initial data. The difficulty to be bypassed in choosing such initial conditions is related to the fact that there is no spectral gap between $\nu_{1}=0$ and the remaining eigenvalues since the compactness of $A$ implies that $\nu_{1}=0$ is an accumulation point of the spectrum, in contrast to finite-dimensional situations. We complete the article with two appendices dealing respectively with some technical question in the proof of Theorem 1 and with a geometric characterization of the eigenvectors of the adjoint of $A$. Aside from its relation to fundamental questions in Stochastic Thermodynamics, this paper was also motivated by the desire to put our analysis into the perspective of the spectral theory of non self-adjoint operators in Hilbert space as developed in [5], which prompted us to choose $l_{\mathbb{C}}^{2}$ as the underlying functional space where our analysis is carried out instead of the geometrically less convenient Banach space $l_{\mathbb{C}}^{1}$ consisting of all absolutely summable complex sequences.

\section{A system of master equations as a dynamical system in $l_{\mathbb{C}}^{2}$}

We write $(., .)_{2}$ for the usual inner product in $l_{\mathbb{C}}^{2}$, which we assume to be linear in the first argument and complex-linear in the second, and $\|\cdot\|_{2}$ for the induced norm. Let us rewrite (3) as

$$
\begin{aligned}
\frac{d p_{\mathrm{m}}(\tau)}{d \tau} & =\sum_{\mathrm{n}=1}^{+\infty} a_{\mathrm{m}, \mathrm{n}} p_{\mathrm{n}}(\tau), \quad \tau \in[0,+\infty), \\
p_{\mathrm{m}}(0) & =p_{\mathrm{m}}^{*}
\end{aligned}
$$

with

$$
a_{\mathrm{m}, \mathrm{n}}=\left\{\begin{array}{c}
-\sum_{\mathrm{k}=1, \mathrm{k} \neq \mathrm{m}}^{+\infty} r_{\mathrm{k}, \mathrm{m}} \quad \text { for } \mathrm{m}=\mathrm{n}, \\
r_{\mathrm{m}, \mathrm{n}} \text { for } \mathrm{m} \neq \mathrm{n} .
\end{array}\right.
$$

Given a sequence $\left(\lambda_{\mathrm{m}}\right)_{\mathrm{m} \in \mathbb{N}^{+}}$of real numbers satisfying (1), we choose the transition rates from level $\mathrm{n}$ to level $\mathrm{m}$ in (6) as

$$
r_{\mathrm{m}, \mathrm{n}}=c_{\mathrm{m}, \mathrm{n}} \exp \left[-\frac{\beta}{2}\left(\lambda_{\mathrm{m}}-\lambda_{\mathrm{n}}\right)\right],
$$

where the prefactors satisfy the symmetry condition $c_{\mathrm{m}, \mathrm{n}}=c_{\mathrm{n}, \mathrm{m}}$ for all $\mathrm{m}, \mathrm{n} \in \mathbb{N}^{+}$ and are otherwise arbitrary, so that the so-called detailed balance conditions

$$
r_{\mathrm{m}, \mathrm{n}} p_{\beta, \mathrm{n}, \text { Gibbs }}=r_{\mathrm{n}, \mathrm{m}} p_{\beta, \mathrm{m}, \text { Gibbs }}
$$


hold. We remark that the choice of (2) as initial data in (5) then provides a time-independent solution to that system of equations, and furthermore that conditions (8) also ensure there is no entropy production in the system under consideration in the sense of the definition proposed in [12] (see also Section II in [13. for more details). It is evidently clear, however, that there are plenty of other choices than (7) which satisfy (8) and for which the results of this article remain valid, but we will keep using (7) mainly for the sake of clarity and simplification. Moreover, in order to make a well-defined dynamical system in $l_{\mathbb{C}}^{2}$ out of (5), we shall use the freedom we have regarding the choice of the $c_{\mathrm{m}, \mathrm{n}}$ by taking

$$
c_{\mathrm{m}, \mathrm{n}}=\exp \left[-\frac{\alpha \beta}{2}\left(\lambda_{\mathrm{m}}+\lambda_{\mathrm{n}}\right)\right]
$$

with $\alpha \in(1,+\infty)$ for all $\mathrm{m}, \mathrm{n} \in \mathbb{N}^{+}$, thereby obtaining the one-parameter family of transition rates

$$
r_{\mathrm{m}, \mathrm{n}}=\exp \left[-\frac{1}{2}\left((\alpha+1) \lambda_{\mathrm{m}}+(\alpha-1) \lambda_{\mathrm{n}}\right) \beta\right]
$$

indexed by $\alpha$. We observe that we then have

$$
\sum_{\mathbf{m}=1}^{+\infty} \sum_{\mathrm{n}=1}^{+\infty} r_{\mathrm{m}, \mathrm{n}}^{2}=Z_{(\alpha-1) \beta} Z_{(\alpha+1) \beta}<+\infty
$$

and

$$
\sum_{\mathrm{m}=1}^{+\infty}\left(\sum_{\mathrm{n}=1}^{+\infty} r_{\mathrm{n}, \mathrm{m}}\right)^{2}=Z_{(\alpha-1) \beta} Z_{\frac{\alpha+1}{2} \beta}^{2}<+\infty
$$

according to (1).

The following preliminary result holds:

Proposition 1. Let us write $\mathrm{p}=\left(p_{\mathrm{m}}\right)_{\mathrm{m} \in \mathbb{N}^{+}}$for any sequence in $l_{\mathbb{C}^{2}}^{2}$. Then the expression

$$
(A \mathrm{p})_{\mathrm{m}}:=\sum_{\mathrm{n}=1}^{+\infty} a_{\mathrm{m}, \mathrm{n}} p_{\mathrm{n}}
$$

defines a linear, non self-adjoint trace-class operator $A: l_{\mathbb{C}}^{2} \mapsto l_{\mathbb{C}}^{2}$ whose trace is given by

$$
\operatorname{Tr} A=Z_{\alpha \beta}-Z_{\frac{\alpha-1}{2} \beta} Z_{\frac{\alpha+1}{2} \beta}<0 .
$$

Proof. From (13) and the Cauchy-Schwarz inequality we get

$$
\|A \mathrm{p}\|_{2}^{2} \leq \sum_{\mathrm{m}=1}^{+\infty} \sum_{\mathrm{n}=1}^{+\infty}\left|a_{\mathrm{m}, \mathrm{n}}\right|^{2} \times\|\mathrm{p}\|_{2}^{2}
$$


where

$$
\begin{aligned}
& \sum_{\mathrm{m}=1}^{+\infty} \sum_{\mathrm{n}=1}^{+\infty}\left|a_{\mathrm{m}, \mathrm{n}}\right|^{2} \\
= & \sum_{\mathrm{m}=1}^{+\infty}\left(\sum_{\mathrm{n}=1, \mathrm{n} \neq \mathrm{m}}^{+\infty} r_{\mathrm{n}, \mathrm{m}}\right)^{2}+\sum_{\mathrm{m}=1}^{+\infty} \sum_{\mathrm{n}=1, \mathrm{n} \neq \mathrm{m}}^{+\infty} r_{\mathrm{m}, \mathrm{n}}^{2} \\
\leq & \sum_{\mathrm{m}=1}^{+\infty}\left(\sum_{\mathrm{n}=1}^{+\infty} r_{\mathrm{n}, \mathrm{m}}\right)^{2}+\sum_{\mathrm{m}=1}^{+\infty} \sum_{\mathrm{n}=1}^{+\infty} r_{\mathrm{m}, \mathrm{n}}^{2} \\
= & Z_{(\alpha-1) \beta}\left(Z_{\frac{\alpha+1}{2} \beta}^{2}+Z_{(\alpha+1) \beta}\right)<+\infty
\end{aligned}
$$

according to (6), (11) and (12), so that $A$ is a linear bounded operator satisfying

$$
\|A \mathrm{p}\|_{2} \leq c(\alpha, \beta)\|\mathrm{p}\|_{2}
$$

for every $\mathrm{p} \in l_{\mathbb{C}}^{2}$ for some $c(\alpha, \beta)>0$. The fact that $A$ is not self-adjoint in general is clear from its definition. Therefore, in order to prove the trace-class property it is necessary and sufficient to show that the series

$$
\sum_{\mathrm{m}=1}^{+\infty}\left(A \mathrm{~h}_{\mathrm{m}}, \mathrm{h}_{\mathrm{m}}\right)_{2}
$$

converges for any orthonormal basis $\left(\mathrm{h}_{\mathrm{m}}\right)_{\mathrm{m} \in \mathbb{N}^{+}}$in $l_{\mathbb{C}}^{2}$, in which case the value of (16) will not depend on the chosen basis (see, e.g., Section 2.3 in Chapter I of [4] or more specifically Theorem 8.1 in Chapter III of [5]). In order to prove convergence we introduce the canonical orthonormal basis in $l_{\mathbb{C}}^{2}$ defined by $\left(\mathrm{e}_{\mathrm{m}}\right)_{\mathrm{n}}=\delta_{\mathrm{m}, \mathrm{n}}$ for all $\mathrm{m}, \mathrm{n} \in \mathbb{N}^{+}$, and expand each $\mathrm{h}_{\mathrm{m}}$ along that basis as

$$
h_{m}=\sum_{n=1}^{+\infty}\left(h_{m}, e_{n}\right)_{2} e_{n}
$$

Then we have

$$
A \mathrm{~h}_{\mathrm{m}}=\sum_{\mathrm{n}=1}^{+\infty}\left(\mathrm{h}_{\mathrm{m}}, \mathrm{e}_{\mathrm{n}}\right)_{2} A \mathrm{e}_{\mathrm{n}}
$$

and therefore

$$
\left(A \mathrm{~h}_{\mathrm{m}}, \mathrm{h}_{\mathrm{m}}\right)_{2}=\sum_{\mathrm{n}=1}^{+\infty} \sum_{\mathrm{k}=1}^{+\infty} a_{\mathrm{k}, \mathrm{n}}\left(\mathrm{h}_{\mathrm{m}}, \mathrm{e}_{\mathrm{n}}\right)_{2}\left(\mathrm{e}_{\mathrm{k}}, \mathrm{h}_{\mathrm{m}}\right)_{2}
$$

so that we get

$$
\begin{aligned}
& \sum_{\mathrm{m}=1}^{+\infty}\left|\left(A \mathrm{~h}_{\mathrm{m}}, \mathrm{h}_{\mathrm{m}}\right)_{2}\right| \leq \frac{1}{2} \sum_{\mathrm{n}=1}^{+\infty} \sum_{\mathrm{k}=1}^{+\infty}\left|a_{\mathrm{k}, \mathrm{n}}\right| \sum_{\mathrm{m}=1}^{+\infty}\left(\left|\left(\mathrm{h}_{\mathrm{m}}, \mathrm{e}_{\mathrm{n}}\right)_{2}\right|^{2}+\left|\left(\mathrm{h}_{\mathrm{m}}, \mathrm{e}_{\mathrm{k}}\right)_{2}\right|^{2}\right) \\
= & \sum_{\mathrm{n}=1}^{+\infty} \sum_{\mathrm{k}=1}^{+\infty}\left|a_{\mathrm{k}, \mathrm{n}}\right| \leq 2 \sum_{\mathrm{n}=1}^{+\infty} \sum_{\mathrm{k}=1}^{+\infty} r_{\mathrm{k}, \mathrm{n}}=2 Z_{\frac{\alpha-1}{2} \beta} Z_{\frac{\alpha+1}{2} \beta}<+\infty
\end{aligned}
$$


since the relation

$$
\sum_{\mathrm{m}=1}^{+\infty}\left|\left(\mathrm{h}_{\mathrm{m}}, \mathrm{e}_{\mathrm{n}}\right)_{2}\right|^{2}=\left\|\mathrm{e}_{\mathrm{n}}\right\|_{2}^{2}=1
$$

holds for every $\mathrm{n} \in \mathbb{N}^{+}$as a consequence of the expansion

$$
e_{n}=\sum_{m=1}^{+\infty}\left(e_{n}, h_{m}\right)_{2} h_{m} .
$$

This proves the desired result with the actual value of the trace given by

$$
\operatorname{Tr} A=\sum_{\mathrm{m}=1}^{+\infty}\left(A \mathrm{e}_{\mathrm{m}}, \mathrm{e}_{\mathrm{m}}\right)_{2}=-\sum_{\mathrm{m}=1}^{+\infty} \sum_{\mathrm{k}=1, \mathrm{k} \neq \mathrm{m}}^{+\infty} r_{\mathrm{k}, \mathrm{m}},
$$

which is (14) as a direct consequence of (10) and (10).

Aside from not being self-adjoint, $A$ is not a normal operator either as $A A^{*} \neq$ $A^{*} A$ in general where $A^{*}$ stands for the adjoint of $A$. Nor is it dissipative as the quadratic form of its imaginary part fails to be positive. However, both $A$ and $A^{*}$ are compact as trace class operators and we shall repeatedly use that property to prove the results which follow. We begin with the following detailed description of some spectral properties:

Theorem 1. Let $A$ be the operator defined by (13). Then the following statements hold:

(a) The spectrum of $A, \sigma(A)$, is a discrete compact set with infinitely many elements $\left(\nu_{\mathrm{k}}\right)_{\mathrm{k} \in \mathbb{N}^{+}}$which are all eigenvalues, including $\nu_{1}=0$.

(b) Assume in addition that $\lambda_{\mathrm{m}+1}>\lambda_{\mathrm{m}}$ for every $\mathrm{m} \in \mathbb{N}^{+}$. Then each eigenvalue $\nu_{\mathrm{k}}$ is implicitly characterized by the relation

$$
\sum_{m=1}^{+\infty} \frac{\exp \left[-\alpha \beta \lambda_{m}\right]}{\nu_{k}+b_{m}}=1
$$

where

$$
b_{\mathrm{m}}:=Z_{\frac{\alpha+1}{2} \beta} \exp \left[-\frac{\alpha-1}{2} \beta \lambda_{\mathrm{m}}\right] .
$$

Moreover each such eigenvalue is real, simple, and the corresponding eigenspace is spanned by $\hat{\mathrm{p}}_{\mathrm{k}}=\left(\hat{p}_{\mathrm{k}, \mathrm{m}}\right)_{\mathrm{m} \in \mathbb{N}^{+}}$where

$$
\hat{p}_{\mathrm{k}, \mathrm{m}}=\frac{\exp \left[-\frac{\alpha+1}{2} \beta \lambda_{\mathrm{m}}\right]}{\nu_{\mathrm{k}}+b_{\mathrm{m}}} .
$$

In particular, the eigenspace associated with $\nu_{1}=0$ is spanned by $\mathrm{p}_{\beta, \text { Gibbs. }}$.

(c) All the non-zero elements of $\sigma(A)$ are negative. More specifically, under the same additional hypothesis as in Statement (b) and if the eigenvalues are 
ordered in such a way that $\nu_{\mathrm{k}}<\nu_{\mathrm{k}+1}$ for every $\mathrm{k} \in\{2,3, \ldots\}$, then we have the localization property $\nu_{\mathrm{k}} \in\left(-b_{\mathrm{k}-1},-b_{\mathrm{k}}\right)$ for every such $\mathrm{k}$.

Proof. The very first part of Statement (a) follows from the fact that $A$ is compact. We also have

$$
A \mathrm{p}_{\beta, \text { Gibbs }}=0
$$

as a consequence of (8) where $\mathrm{p}_{\beta, \text { Gibbs }} \in l_{\mathbb{C}}^{2}$, so that $\nu_{1}=0$ is indeed an eigenvalue.

As for the proof of Statement (b), let us take $p \in l_{\mathbb{C}}^{2}$ with $\mathrm{p} \neq 0$ and

$$
A \mathrm{p}=\nu_{\mathrm{k}} \mathrm{p}
$$

Owing to (6) and (13), this is equivalent to requiring that

$$
\sum_{\mathrm{n}=1}^{+\infty} r_{\mathrm{m}, \mathrm{n}} p_{\mathrm{n}}-p_{\mathrm{m}} \sum_{\mathrm{n}=1}^{+\infty} r_{\mathrm{n}, \mathrm{m}}=\nu_{\mathrm{k}} p_{\mathrm{m}}
$$

for every $m \in \mathbb{N}^{+}$. Therefore, using (10) we obtain

$$
c_{\mathrm{p}, \alpha, \beta} \exp \left[-\frac{\alpha+1}{2} \beta \lambda_{\mathrm{m}}\right]=\left(\nu_{\mathrm{k}}+b_{\mathrm{m}}\right) p_{\mathrm{m}}
$$

for every $\mathrm{m}$ after some rearrangements, where $b_{\mathrm{m}}$ is given by (18) and

$$
c_{\mathrm{p}, \alpha, \beta}:=\sum_{\mathrm{n}=1}^{+\infty} \exp \left[-\frac{\alpha-1}{2} \beta \lambda_{\mathrm{n}}\right] p_{\mathrm{n}} .
$$

Consequently, if $c_{\mathrm{p}, \alpha, \beta} \neq 0$ then $\nu_{\mathrm{k}}+b_{\mathrm{m}} \neq 0$ for every $\mathrm{m}$ so that (20) leads to

$$
\mathrm{p}=c_{\mathrm{p}, \alpha, \beta} \hat{\mathrm{p}}_{\mathrm{k}},
$$

and we claim that this is the only possible case. Indeed, on the one hand if $c_{\mathrm{p}, \alpha, \beta}=0$ then $\left(\nu_{\mathrm{k}}+b_{\mathrm{m}}\right) p_{\mathrm{m}}=0$ for every $\mathrm{m}$. But on the other hand, since $\mathrm{m} \neq \mathrm{n}$ implies $\lambda_{\mathrm{m}} \neq \lambda_{\mathrm{n}}$ we have $b_{\mathrm{m}} \neq b_{\mathrm{n}}$, so that there may exist at most one $\mathrm{m}^{*}$ with $\nu_{\mathrm{k}}+b_{\mathrm{m}^{*}}=0$. If there is no such $\mathrm{m}^{*}$ then we must have $p_{\mathrm{m}}=0$ for every $m$, hence $p=0$ which is not an eigenvector. If there is such an $m^{*}$ then $p_{\mathrm{m}}=0$ for every $\mathrm{m} \neq \mathrm{m}^{*}$ but we may have $p_{\mathrm{m}^{*}}=0$ or $p_{\mathrm{m}^{*}} \neq 0$. In the first case we get $p=0$ once again, while in the second case (21) reduces to

$$
c_{\mathrm{p}, \alpha, \beta}=\exp \left[-\frac{\alpha-1}{2} \beta \lambda_{\mathrm{m}^{*}}\right] p_{\mathrm{m}^{*}} \neq 0,
$$

a contradiction. Therefore, the eigenspace associated with $\nu_{\mathrm{k}}$ is indeed the one-dimensional subspace generated by $\hat{p}_{\mathrm{k}}$. The fact that $\hat{p}_{\mathrm{k}} \in l_{\mathbb{C}}^{2}$ is a simple consequence of (1) which is easily verified in case of $\mathrm{p}_{\beta, \text { Gibbs }}$, while for $\nu_{\mathrm{k}} \neq 0$ we have

$$
\sum_{\mathrm{m}=1}^{+\infty}\left|\nu_{\mathrm{k}}+b_{\mathrm{m}}\right|^{2}\left|\hat{p}_{\mathrm{k}, \mathrm{m}}\right|^{2}=Z_{(\alpha+1) \beta}<+\infty
$$


from (11) and (19), which implies $\sum_{\mathrm{m}=1}^{+\infty}\left|\hat{p}_{\mathrm{k}, \mathrm{m}}\right|^{2}<+\infty$ by asymptotic comparison since $b_{\mathrm{m}} \rightarrow 0$ as $\mathrm{m} \rightarrow+\infty$. Finally, the substitution of (22) into (21) using (19) gives (17) whose imaginary part is then equal to zero, that is,

$$
\sum_{\mathrm{m}=1}^{+\infty} \operatorname{Im} \frac{\exp \left[-\alpha \beta \lambda_{\mathrm{m}}\right]}{\nu_{\mathrm{k}}+b_{\mathrm{m}}}=-\operatorname{Im} \nu_{\mathrm{k}} \sum_{\mathrm{m}=1}^{+\infty} \frac{\exp \left[-\alpha \beta \lambda_{\mathrm{m}}\right]}{\left|\nu_{\mathrm{k}}+b_{\mathrm{m}}\right|^{2}}=0
$$

which implies that each $\nu_{\mathrm{k}}$ is real.

As for Statement (c), let us first prove that $\nu_{\mathrm{k}}<0$ for every non-zero $\nu_{\mathrm{k}} \in$ $\sigma(A)$. Since $\nu_{\mathrm{k}}$ is real the adjoint equation reads

$$
A^{*} \mathrm{q}=\nu_{\mathrm{k}} \mathrm{q}
$$

for some $\mathrm{q} \in l_{\mathbb{C}}^{2}$ with $\mathrm{q} \neq 0$. Since $\mathrm{q} \in l_{\mathbb{C}}^{2}$ implies that $q_{\mathrm{m}} \rightarrow 0$ as $\mathrm{m} \rightarrow+\infty$ and since $\mathbf{q} \neq 0$, there exists $\mathrm{m}^{*} \in \mathbb{N}^{+}$such that $\left|q_{\mathrm{m}^{*}}\right|>0$ and $\left|q_{\mathrm{m}}\right| \leqslant\left|q_{\mathrm{m}^{*}}\right|$ for every $\mathrm{m}$. Consequently, noting that (23) implies in particular the relation

$$
\left(\nu_{\mathrm{k}}-a_{\mathrm{m}^{*}, \mathrm{~m}^{*}}\right) q_{\mathrm{m}^{*}}=\sum_{\mathrm{n}=1, \mathrm{n} \neq \mathrm{m}^{*}}^{+\infty} r_{\mathrm{n}, \mathrm{m}^{*}} q_{\mathrm{n}}
$$

according to (6), we get after simplification

$$
\left|\left(\nu_{\mathrm{k}}-a_{\mathrm{m}^{*}, \mathrm{~m}^{*}}\right)\right| \leqslant \sum_{\mathrm{n}=1, \mathrm{n} \neq \mathrm{m}^{*}}^{+\infty} r_{\mathrm{n}, \mathrm{m}^{*}}=\left|a_{\mathrm{m}^{*}, \mathrm{~m}^{*}}\right|
$$

or, equivalently,

$$
\nu_{\mathrm{k}}^{2}-2 a_{\mathrm{m}^{*}, \mathrm{~m}^{*}} \nu_{\mathrm{k}} \leqslant 0
$$

where $\nu_{\mathrm{k}}^{2}>0$ since $\nu_{\mathrm{k}} \neq 0$, and therefore $\nu_{\mathrm{k}}<0$ because $a_{\mathrm{m}^{*}, \mathrm{~m}^{*}}<0$. More specifically, from our hypothesis regarding $\lambda_{\mathrm{m}}$ we get $b_{\mathrm{m}}>b_{\mathrm{m}+1}$ from (18) for every $\mathrm{m} \in \mathbb{N}^{+}$. Then we have $\nu_{\mathrm{k}} \in\left(-b_{1}, 0\right)$ for every $\mathrm{k} \in\{2,3, \ldots\}$, for if there were at least one $\mathrm{k}^{*}$ with $\nu_{\mathrm{k}^{*}} \notin\left(-b_{1}, 0\right)$ we would necessarily have $\nu_{\mathrm{k}^{*}}+b_{\mathrm{m}} \leqslant 0$ for each $\mathrm{m}$, thereby contradicting (17). Now, let us consider the function $f:(-\infty, 0) \backslash\left\{-b_{\mathrm{m}}, \mathrm{m} \in \mathbb{N}^{+}\right\} \mapsto \mathbb{R}$ given by

$$
f(\nu):=\sum_{\mathrm{m}=1}^{+\infty} \frac{\exp \left[-\alpha \beta \lambda_{\mathrm{m}}\right]}{\nu+b_{\mathrm{m}}} .
$$

Since $b_{\mathrm{m}} \rightarrow 0$ as $\mathrm{m} \rightarrow+\infty$ it is plain that this series converges absolutely by asymptotic comparison and by virtue of (1). Moreover, as a consequence of the properties of the holomorphic continuation of (25) investigated in Appendix A, we have

$$
\begin{aligned}
\lim _{\nu \searrow-b_{\mathrm{k}-1}} f(\nu) & =+\infty, \\
\lim _{\nu \nearrow-b_{\mathrm{k}}} f(\nu) & =-\infty
\end{aligned}
$$


and $f^{\prime}(\nu)<0$ whenever $\nu \in\left(-b_{\mathrm{k}-1},-b_{\mathrm{k}}\right)$, so that in particular there exists a unique $\nu^{*} \in\left(-b_{\mathrm{k}-1},-b_{\mathrm{k}}\right)$ with $f\left(\nu^{*}\right)=1$. Therefore we necessarily have $\nu^{*}=\nu_{\mathrm{k}}$ as a consequence of (17), which proves the desired localization property. Finally we have $f(0):=\lim _{\nu} \nearrow_{0} f(\nu)=1$, which is yet another way of saying that (17) also characterizes $\nu_{1}=0$.

Remarks. (1) The eigenvectors of $A^{*}$ in (23) corresponding to $\nu_{\mathrm{k}} \neq 0$ can also be determined by using the method that led to (19). Each one of them is indeed a multiple of $\hat{q}_{k}$ whose components are

$$
\hat{q}_{\mathrm{k}, \mathrm{m}}=\frac{\exp \left[-\frac{\alpha-1}{2} \beta \lambda_{\mathrm{m}}\right]}{\nu_{\mathrm{k}}+b_{\mathrm{m}}}
$$

for each $\mathrm{k} \in\{2,3, \ldots\}$ and every $\mathrm{m} \in \mathbb{N}^{+}$. Moreover, in contrast to $A$ it is interesting to note that $\nu_{1}=0 \in \sigma\left(A^{*}\right)$ is not an eigenvalue.

(2) Whereas (17) holds for all the eigenvalues of $A$, the relation

$$
\sum_{\mathrm{m}=1}^{+\infty} \frac{\exp \left[-\frac{\alpha+1}{2} \beta \lambda_{\mathrm{m}}\right]}{\nu_{\mathrm{k}}+b_{\mathrm{m}}}=0
$$

only characterizes the non-zero eigenvalues, that is, holds for each $k \in\{2,3, \ldots\}$. Indeed for every such $\mathrm{k}$ we have

$$
\begin{aligned}
0 & =f\left(\nu_{\mathrm{k}}\right)-f(0) \\
& =-\nu_{\mathrm{k}} \sum_{\mathrm{m}=1}^{+\infty} \frac{\exp \left[-\alpha \beta \lambda_{\mathrm{m}}\right]}{b_{\mathrm{m}}\left(\nu_{\mathrm{k}}+b_{\mathrm{m}}\right)} \\
& =-\frac{\nu_{\mathrm{k}}}{Z_{\frac{\alpha+1}{2} \beta}} \sum_{\mathrm{m}=1}^{+\infty} \frac{\exp \left[-\frac{\alpha+1}{2} \beta \lambda_{\mathrm{m}}\right]}{\nu_{\mathrm{k}}+b_{\mathrm{m}}}
\end{aligned}
$$

from (17), (18) and (25), which leads to (27) since $\nu_{\mathrm{k}} \neq 0$. Relation (27) will play an important role later on when we prove that the $\hat{p}_{\mathrm{k}}$ constitute a basis of $l_{\mathbb{C}}^{2}$ under a more stringent condition regarding the sequence $\left(\lambda_{\mathrm{m}}\right)_{\mathrm{m} \in \mathbb{N}^{+}}$, a fact that will lead to the spectral decomposition of the semigroup generated by $A$ and thereby to its ultimate behavior for large times.

For now our objective is to prove the completeness of the $\hat{p}_{\mathrm{k}}$ in the sense that the set of all their finite linear combinations is everywhere dense in $l_{\mathbb{C}}^{2}$, provided we impose an additional restriction on the parameter $\alpha$. Our preliminary step in that direction is to rule out the existence of root vectors of height larger than one. Let us recall that a root vector $\mathrm{q} \in l_{\mathbb{C}}^{2}, \mathrm{q} \neq 0$, associated with the eigenvalue $\nu_{\mathrm{k}}$ of $A$ is one that satisfies

$$
\left(A-\nu_{\mathrm{k}}\right)^{\mathrm{n}} \mathrm{q}=0
$$

for some $\mathrm{n} \in \mathbb{N}^{+}$, and that the height $\mathfrak{h}(\mathbf{q})$ of $\mathbf{q}$ is the least integer for which the preceding relation holds (see, e.g., Chapter 6 in [16 for a general definition). The precise result is the following: 
Proposition 2. Let us assume that $\lambda_{\mathrm{m}+1}>\lambda_{\mathrm{m}}$ for every $\mathrm{m} \in \mathbb{N}^{+}$. Then we have

$$
\operatorname{ker}\left(A-\nu_{\mathrm{k}}\right)^{\mathrm{n}}=\operatorname{ker}\left(A-\nu_{\mathrm{k}}\right)=\operatorname{span}\left\{\hat{\mathrm{p}}_{\mathrm{k}}\right\}
$$

for every $\mathrm{n} \in \mathbb{N}^{+}$and every $\mathrm{k} \in \mathbb{N}^{+}$, where $\hat{\mathrm{p}}_{\mathrm{k}}$ is given by (19). Thus $\mathfrak{h}(\mathbf{q})=1$ for every root vector of $A$.

Proof. The statement is true for $\mathrm{n}=1$ according to (b) of Theorem 1, so that we proceed by induction on $\mathrm{n}$. Assuming then that (28) holds we take $\mathrm{q} \in \operatorname{ker}\left(A-\nu_{\mathrm{k}}\right)^{\mathrm{n}+1}$, which gives

$$
\left(A-\nu_{\mathrm{k}}\right)^{\mathrm{n}+1} \mathrm{q}=\left(A-\nu_{\mathrm{k}}\right)^{\mathrm{n}}\left(A-\nu_{\mathrm{k}}\right) \mathrm{q}=0
$$

and hence

$$
\left(A-\nu_{\mathrm{k}}\right) \mathrm{q}=\gamma \hat{\mathrm{p}}_{\mathrm{k}}
$$

for some $\gamma \in \mathbb{C}$. We proceed to show that $\gamma=0$ is the only value for which (29) may hold by proving that there is no $\mathrm{q} \in l_{\mathbb{C}}^{2}$ with

$$
\left(A-\nu_{\mathrm{k}}\right) \mathrm{q}=\hat{\mathrm{p}}_{\mathrm{k}} .
$$

Arguing indirectly and using the same method as in the proof of Theorem 1 we see that (30) is equivalent to having

$$
q_{\mathrm{k}, \mathrm{m}}=c_{\mathrm{q}, \alpha, \beta} \hat{p}_{\mathrm{k}, \mathrm{m}}-\frac{\hat{p}_{\mathrm{k}, \mathrm{m}}}{\nu_{\mathrm{k}}+b_{\mathrm{m}}}
$$

for every $m \in \mathbb{N}^{+}$, where we used (19) and

$$
c_{\mathrm{q}, \alpha, \beta}:=\sum_{\mathrm{n}=1}^{+\infty} \exp \left[-\frac{\alpha-1}{2} \beta \lambda_{\mathrm{n}}\right] q_{\mathrm{n}} .
$$

If such a q were to provide a solution to (30), its components (31) should be compatible with $c_{\mathrm{q}, \alpha, \beta}$ given by (32). But the substitution of (31) into (32) and a repeated use of (19) along with (17) show that compatibility is possible if, and only if,

$$
\sum_{\mathrm{m}=1}^{+\infty} \frac{\exp \left[-\alpha \beta \lambda_{\mathrm{m}}\right]}{\left(\nu_{\mathrm{k}}+b_{\mathrm{m}}\right)^{2}}=0
$$

for every $k \in \mathbb{N}^{+}$, which contradicts the fact that each term of the preceding expression is positive. Therefore (29) is only valid with $\gamma=0$ so that

$$
\operatorname{ker}\left(A-\nu_{\mathrm{k}}\right)^{\mathrm{n}+1} \subseteq \operatorname{ker}\left(A-\nu_{\mathrm{k}}\right),
$$

which proves the desired result since the converse inclusion is trivial.

REMARK. With an identical proof we get a similar result for $A^{*}$, namely,

$$
\operatorname{ker}\left(A^{*}-\nu_{\mathrm{k}}\right)^{\mathrm{n}}=\operatorname{ker}\left(A^{*}-\nu_{\mathrm{k}}\right)=\operatorname{span}\left\{\hat{\mathrm{q}}_{\mathrm{k}}\right\}
$$


for every $\mathrm{n} \in \mathbb{N}^{+}$and every $\mathrm{k} \in\{2,3, \ldots\}$, where $\hat{\mathrm{q}}_{\mathrm{k}}$ is given by (26).

There are many known and well-documented criteria that ensure the completeness of the root vectors of a given non self-adjoint operator on a Hilbert space, and thereby the possibility of constructing a basis consisting of such vectors (see, e.g., Chapters V and VI in [5]). As far as $A$ is concerned we shall settle for an application of a theorem that originally appeared in [8], which is stated and proved as Theorem 8.1 in Chapter V of [5]. Our application, however, will be very indirect given the fact that the theorem in question requires the operator under investigation to have a trivial kernel, a property not shared by the operator $A$. Nevertheless, we will now show that we can bypass this difficulty by means of an auxiliary operator that has the desired properties. Let us denote by

$$
P: l_{\mathbb{C}}^{2} \mapsto \operatorname{ker} A
$$

the orthogonal projection onto the eigenspace generated by $\mathrm{p}_{\beta, \text { Gibbs }}$, and let $\mathrm{us}$ consider the compact operator $A+P$ whose eigenvalues we denote by $\left(\varkappa_{\mathrm{k}}\right)_{\mathrm{k}=1}^{+\infty}$. In the sequel and for the sake of convenience we write $E_{\nu_{\mathrm{k}}}(A)$ for the eigenspace of the operator $A$ associated with the eigenvalue $\nu_{\mathrm{k}}, E_{\varkappa_{\mathrm{k}}}(A+P)$ for that of the operator $A+P$ associated with the eigenvalue $\varkappa_{k}$ and

$$
R_{\varkappa_{\mathrm{k}}}(A+P)=\bigcup_{\mathrm{n}=1}^{+\infty} \operatorname{ker}\left(A+P-\varkappa_{\mathrm{k}}\right)^{\mathrm{n}}
$$

for the corresponding root vector subspace.

We have the following preliminary result:

Proposition 3. Let us assume that $\lambda_{\mathrm{m}+1}>\lambda_{\mathrm{m}}$ for every $\mathrm{m} \in \mathbb{N}^{+}$. Then we have

$$
\operatorname{ker}(A+P)=\{0\}
$$

and

$$
\bigvee_{\mathrm{k}=1}^{+\infty} E_{\nu_{\mathrm{k}}}(A)=\bigvee_{\mathrm{k}=1}^{+\infty} R_{\varkappa_{\mathrm{k}}}(A+P),
$$

where the spaces in (35) stand for the closed linear hull of $\cup_{\mathrm{k}=1}^{+\infty} E_{\nu_{\mathrm{k}}}(A)$ and $\cup_{\mathrm{k}=1}^{+\infty} R_{\varkappa_{\mathrm{k}}}(A+P)$, respectively.

Proof. If $\mathrm{q} \in \operatorname{ker}(A+P)$ we have

$$
A \mathrm{q}=-\left(\mathrm{q}, \hat{\mathrm{p}}_{\beta, \text { Gibbs }}\right)_{2} \hat{\mathrm{p}}_{\beta, \text { Gibbs }}
$$

where $\hat{\mathbf{p}}_{\beta, \text { Gibbs }}$ now stands for $\mathbf{p}_{\beta, \text { Gibbs }}$ renormalized in such a way that $\left\|\hat{\mathbf{p}}_{\beta, \text { Gibbs }}\right\|_{2}=$ 1 , and therefore $\mathrm{q} \in E_{\nu_{1}=0}(A)^{\perp}$ as a consequence of the non existence result of a $q$ satisfying (29) when $\gamma \neq 0$. On the other hand we infer from (36) that $A^{2} \mathrm{q}=0$, which implies $\mathrm{q} \in E_{\nu_{1}=0}(A)$ by virtue of Proposition 2 and thereby $\mathrm{q} \in E_{\nu_{1}=0}(A) \cap E_{\nu_{1}=0}(A)^{\perp}=\{0\}$, which proves (34). 
Next, we show that

$$
\bigvee_{\mathrm{k}=1}^{+\infty} E_{\nu_{\mathrm{k}}}(A) \subseteq \bigvee_{\mathrm{k}=1}^{+\infty} R_{\varkappa_{\mathrm{k}}}(A+P)
$$

We first have

$$
E_{\nu_{1}=0}(A) \subseteq \bigvee_{\mathrm{k}=1}^{+\infty} R_{\varkappa_{\mathrm{k}}}(A+P)
$$

since $\hat{\mathbf{p}}_{\beta, \text { Gibbs }}$ is also an eigenvector of $A+P$ with eigenvalue $\varkappa=1$, that is,

$$
(A+P) \hat{\mathbf{p}}_{\beta, \text { Gibbs }}=\hat{\mathbf{p}}_{\beta, \text { Gibbs }} .
$$

Moreover, all the non zero eigenvalues of $A$ are eigenvalues of $A+P$ for some suitably constructed eigenvector. Indeed, for each $\mathrm{k} \in\{2,3 \ldots\}$ let us pick an arbitrary $\mathrm{q}_{\mathrm{k}} \in E_{\nu_{\mathrm{k}}}(A)$ with $\mathrm{q}_{\mathrm{k}} \neq 0$, and let $\mathrm{q}_{\mathrm{k}, \gamma}=\mathrm{q}_{\mathrm{k}}+\gamma \hat{\mathbf{p}}_{\beta, \text { Gibbs }}$ for $\gamma \in \mathbb{C}$. Using the properties of $A$ and $P$ already established we then get

$$
\left(A+P-\nu_{\mathrm{k}}\right) \mathrm{q}_{\mathrm{k}, \gamma}=P \mathrm{q}_{\mathrm{k}}+\gamma\left(1-\nu_{\mathrm{k}}\right) \hat{\mathrm{p}}_{\beta, \text { Gibbs }}
$$

where

$$
P \mathrm{q}_{\mathrm{k}}=\left(\mathrm{q}_{\mathrm{k}}, \hat{\mathrm{p}}_{\beta, \text { Gibbs }}\right)_{2} \hat{\mathrm{p}}_{\beta, \text { Gibbs }}
$$

Thus

$$
\left(A+P-\nu_{\mathrm{k}}\right) \mathrm{q}_{\mathrm{k}, \gamma}=0
$$

if, and only if,

$$
\left(\left(q_{\mathrm{k}}, \hat{\mathbf{p}}_{\beta, \text { Gibbs }}\right)_{2}+\gamma\left(1-\nu_{\mathrm{k}}\right)\right) \hat{\mathbf{p}}_{\beta, \text { Gibbs }}=0 .
$$

But according to Statement (b) of Theorem 1 we have $\nu_{\mathrm{k}} \neq 1$ for every $\mathrm{k}$, so that the choice of

$$
\gamma_{\mathrm{k}}=\frac{\left(\mathrm{q}_{\mathrm{k}}, \hat{\mathrm{p}}_{\beta, \text { Gibbs }}\right)_{2}}{\nu_{\mathrm{k}}-1}
$$

leads to $\hat{\mathrm{q}}_{\mathrm{k}}:=\mathrm{q}_{\mathrm{k}}+\gamma_{\mathrm{k}} \hat{\mathrm{p}}_{\beta \text {, Gibbs }} \in E_{\nu_{\mathrm{k}}}(A+P)$ with $\hat{\mathrm{q}}_{\mathrm{k}} \neq 0$. Indeed, $\hat{\mathrm{q}}_{\mathrm{k}}=0$ would imply $\mathrm{q}_{\mathrm{k}} \in E_{\nu_{1}=0}(A)$, a contradiction. Consequently, because of (39) we have both $\hat{\mathrm{q}}_{\mathrm{k}}, \hat{\mathrm{p}}_{\beta, \text { Gibbs }} \in \cup_{\mathrm{k}=1}^{+\infty} R_{\varkappa_{\mathrm{k}}}(A+P)$, of which $\mathrm{q}_{\mathrm{k}}=\hat{\mathrm{q}}_{\mathrm{k}}-\gamma_{\mathrm{k}} \hat{\mathrm{p}}_{\beta, \text { Gibbs }}$ is a linear combination. This along with (38) proves that

$$
E_{\nu_{\mathrm{k}}}(A) \subseteq \bigvee_{\mathrm{k}=1}^{+\infty} R_{\varkappa_{\mathrm{k}}}(A+P)
$$

for every $k \in \mathbb{N}^{+}$, from which (37) follows.

In order to prove the converse inclusion we first observe that

$$
E_{\varkappa=1}(A+P) \subseteq \bigvee_{\mathrm{k}=1}^{+\infty} E_{\nu_{\mathrm{k}}}(A)
$$


It follows indeed from the projection theorem in Hilbert space that

$$
E_{\varkappa=1}(A+P)=E_{\nu_{1}=0}(A)
$$

for the relation $(A+P-1) \mathbf{q}=0$ with $\mathbf{q}=\gamma \hat{\mathbf{p}}_{\beta, \text { Gibbs }}+\hat{\mathrm{q}}$ for some $\gamma \in \mathbb{C}$ and $\left(\hat{\mathrm{q}}, \hat{\mathrm{p}}_{\beta, \text { Gibbs }}\right)_{2}=0$ implies that $A \hat{\mathrm{q}}=\hat{\mathrm{q}}$, hence that $\hat{\mathrm{q}}=0$ since $\nu=1$ is not an eigenvalue of $A$, which leads to (40).

For the other eigenvalues of $A+P$ let us take an arbitray $\mathrm{q}_{\mathrm{k}} \in R_{\varkappa_{\mathrm{k}}}(A+P)$. Then there exists $\mathrm{n}^{*} \in \mathbb{N}^{+}$such that

$$
\left(A+P-\varkappa_{\mathrm{k}}\right)^{\mathrm{n}^{*}} \mathrm{q}_{\mathrm{k}}=0 .
$$

Moreover, since $A P=0$ we have the operator equality

$$
\left(A+P-\varkappa_{\mathrm{k}}\right)^{\mathrm{n}}=\left(A-\varkappa_{\mathrm{k}}\right)^{\mathrm{n}}+P \sum_{\mathrm{j}=0}^{\mathrm{n}-1}\left(1-\varkappa_{\mathrm{k}}\right)^{\mathrm{n}-1-\mathrm{j}}\left(A-\varkappa_{\mathrm{k}}\right)^{\mathrm{j}}
$$

valid for every $\mathrm{n} \in \mathbb{N}^{+}$, which follows from an easy induction argument. We then proceed as in the first part of the proof by considering $\mathbf{q}_{\mathrm{k}, \gamma}=\mathbf{q}_{\mathrm{k}}+\gamma \hat{\mathbf{p}}_{\beta, \text { Gibbs }}$ for $\gamma \in \mathbb{C}$, and by determining the result of the action of both sides of (42) on $\mathrm{q}_{\mathrm{k}, \gamma}$ when $\mathrm{n}=\mathrm{n}^{*}$. For the left-hand side we have

$$
\left(A+P-\varkappa_{\mathrm{k}}\right)^{\mathrm{n}^{*}} \mathrm{q}_{\mathrm{k}, \gamma}=\gamma\left(1-\varkappa_{\mathrm{k}}\right)^{\mathrm{n}^{*}} \hat{\mathrm{p}}_{\beta, \text { Gibbs }}
$$

as a consequence of (41), while for the right-hand side we may write

$$
\begin{aligned}
& \left(A-\varkappa_{\mathrm{k}}\right)^{\mathrm{n}^{*}} \mathrm{q}_{\mathrm{k}, \gamma}+P \sum_{\mathrm{j}=0}^{\mathrm{n}^{*}-1}\left(1-\varkappa_{\mathrm{k}}\right)^{\mathrm{n}^{*}-1-\mathrm{j}}\left(A-\varkappa_{\mathrm{k}}\right)^{\mathrm{j}} \mathrm{q}_{\mathrm{k}, \gamma} \\
= & \left(A-\varkappa_{\mathrm{k}}\right)^{\mathrm{n}^{*}} \mathrm{q}_{\mathrm{k}, \gamma}+P \sum_{\mathrm{j}=0}^{\mathrm{n}^{*}-1}\left(1-\varkappa_{\mathrm{k}}\right)^{\mathrm{n}^{*}-1-\mathrm{j}}\left(A-\varkappa_{\mathrm{k}}\right)^{\mathrm{j}} \mathrm{q}_{\mathrm{k}} \\
+ & \gamma \sum_{\mathrm{j}=0}^{\mathrm{n}^{*}-1}\left(1-\varkappa_{\mathrm{k}}\right)^{\mathrm{n}^{*}-1-\mathrm{j}}\left(-\varkappa_{\mathrm{k}}\right)^{\mathrm{j}} \hat{\mathrm{p}}_{\beta, \text { Gibbs }}
\end{aligned}
$$

since

$$
P\left(A-\varkappa_{\mathrm{k}}\right)^{\mathrm{j}} \hat{\mathrm{p}}_{\beta, \text { Gibbs }}=\left(-\varkappa_{\mathrm{k}}\right)^{\mathrm{j}} \hat{\mathrm{p}}_{\beta, \text { Gibbs }}
$$

for every j. But (43) and (44) are equal, so that by regrouping and rearranging terms we obtain

$$
\left(A-\varkappa_{\mathrm{k}}\right)^{\mathrm{n}^{*}} \mathrm{q}_{\mathrm{k}, \gamma}=\gamma\left(-\varkappa_{\mathrm{k}}\right)^{\mathrm{n}^{*}} \hat{\mathrm{p}}_{\beta, \text { Gibbs }}-P \sum_{\mathrm{j}=0}^{\mathrm{n}^{*}-1}\left(1-\varkappa_{\mathrm{k}}\right)^{\mathrm{n}^{*}-1-\mathrm{j}}\left(A-\varkappa_{\mathrm{k}}\right)^{\mathrm{j}} \mathrm{q}_{\mathrm{k}}
$$

where we have used the identity

$$
\sum_{j=0}^{n-1}\left(1-\varkappa_{k}\right)^{n-1-j}\left(-\varkappa_{k}\right)^{j}-\left(1-\varkappa_{k}\right)^{n}=-\left(-\varkappa_{k}\right)^{n}
$$


valid for every $n \in \mathbb{N}^{+}$. Thus we have

$$
\left(A-\varkappa_{\mathrm{k}}\right)^{\mathrm{n}^{*}} \mathrm{q}_{\mathrm{k}, \gamma}=0
$$

if, and only if,

$$
\gamma\left(-\varkappa_{\mathrm{k}}\right)^{\mathrm{n}^{*}} \hat{\mathrm{p}}_{\beta, \text { Gibbs }}=P \sum_{\mathrm{j}=0}^{\mathrm{n}^{*}-1}\left(1-\varkappa_{\mathrm{k}}\right)^{\mathrm{n}^{*}-1-\mathrm{j}}\left(A-\varkappa_{\mathrm{k}}\right)^{\mathrm{j}} \mathrm{q}_{\mathrm{k}} .
$$

But according to (34) we have $\varkappa_{k} \neq 0$ for every $k$, so that we may choose

$$
\gamma_{\mathrm{k}}=\left(-\varkappa_{\mathrm{k}}\right)^{-\mathrm{n}^{*}}\left(P \sum_{\mathrm{j}=0}^{\mathrm{n}^{*}-1}\left(1-\varkappa_{\mathrm{k}}\right)^{\mathrm{n}^{*}-1-\mathrm{j}}\left(A-\varkappa_{\mathrm{k}}\right)^{\mathrm{j}} \mathrm{q}_{\mathrm{k}}, \hat{\mathrm{p}}_{\beta, \text { Gibbs }}\right)_{2}
$$

to have $\hat{\mathrm{q}}_{\mathrm{k}}:=\mathrm{q}_{\mathrm{k}}+\gamma_{\mathrm{k}} \hat{\mathrm{p}}_{\beta}$, Gibbs $\in \operatorname{ker}\left(A-\varkappa_{\mathrm{k}}\right)^{\mathrm{n}^{*}}$ with $\hat{\mathrm{q}}_{\mathrm{k}} \neq 0$, hence $\hat{\mathrm{q}}_{\mathrm{k}} \in E_{\varkappa_{\mathrm{k}}}(A)$ according to Proposition 2. Arguing then as in the first part of the proof and taking (40) into account we obtain

$$
\bigvee_{\mathrm{k}=1}^{+\infty} R_{\varkappa \mathrm{k}}(A+P) \subseteq \bigvee_{\mathrm{k}=1}^{+\infty} E_{\nu_{\mathrm{k}}}(A)
$$

The preceding considerations now lead to the following result:

Theorem 2. Let us assume that $\lambda_{\mathrm{m}+1}>\lambda_{\mathrm{m}}$ for every $\mathrm{m} \in \mathbb{N}^{+}$, and let us impose the additional restriction $\alpha \in(1,3)$ on the parameter introduced in (9). Then the set of all $\hat{\mathrm{p}}_{\mathrm{k}}$ is complete in $l_{\mathbb{C}}^{2}$, that is,

$$
l_{\mathbb{C}}^{2}=\bigvee_{\mathrm{k}=1}^{+\infty} E_{\nu_{\mathrm{k}}}(A)
$$

Proof. According to (35) it is sufficient to show that the set of all root vectors of the operator $A+P$ is complete in $l_{\mathbb{C}}^{2}$. To this end we realize $A+P$ as a perturbation of a linear, bounded, invertible self-adjoint operator $H$ of finite order in the sense of Theorem 8.1 in Chapter V of [5], namely,

$$
A+P=H\left(I+S+H^{-1} P\right)
$$

where $I$ stands for the identity operator in $l_{\mathbb{C}}^{2}, S$ for a linear compact operator and $H^{-1} P$ compact. In order to achieve that we define $H$ and $S$ by their matrix elements

$$
h_{\mathrm{m}, \mathrm{n}}:=-b_{\mathrm{m}} \delta_{\mathrm{m}, \mathrm{n}}
$$

and

$$
s_{\mathrm{m}, \mathrm{n}}:=-\frac{r_{\mathrm{m}, \mathrm{n}}}{b_{\mathrm{m}}}
$$


for all $\mathrm{m}, \mathrm{n} \in \mathbb{N}^{+}$, respectively, where $r_{\mathrm{m}, \mathrm{n}}$ is given by (7) and $b_{\mathrm{m}}$ by (18). It is easily verified from (47) that $H$ is trace-class and thereby of finite order, the other required properties of $H$ being obvious. Using arguments similar to those invoked in the proof of Proposition 1, it is equally straightforward to check that $S$ is also trace-class and thereby compact. As for $H^{-1} P$ we have

$$
H^{-1} P \mathrm{q}=\left(\mathbf{q}, \hat{\mathrm{p}}_{\beta, \text { Gibbs }}\right)_{2} H^{-1} \hat{\mathrm{p}}_{\beta, \text { Gibbs }}
$$

for every $\mathrm{q} \in l_{\mathbb{C}}^{2}$, where

$$
\left(H^{-1} \hat{\mathbf{p}}_{\beta, \text { Gibbs }}\right)_{\mathrm{m}}=-\frac{\hat{p}_{\beta, \text { Gibbs }, \mathrm{m}}}{b_{\mathrm{m}}}=c_{\alpha, \beta} \exp \left[-\frac{3-\alpha}{2} \beta \lambda_{\mathrm{m}}\right]
$$

for each $\mathrm{m} \in \mathbb{N}^{+}$and some irrelevant constant $c_{\alpha, \beta} \in \mathbb{R}$. But we have assumed $\alpha \in(1,3)$ and therefore, changing the value of $c_{\alpha, \beta}$ whenever necessary, we obtain

$$
H^{-1} \hat{\mathbf{p}}_{\beta, \text { Gibbs }}=c_{\alpha, \beta} \hat{\mathbf{p}}_{\frac{3-\alpha}{2} \beta, \text { Gibbs }}
$$

so that $H^{-1} P$ turns out to be a bounded operator of rank one in $l_{\mathbb{C}}^{2}$ and thereby also compact. Finally, using (6), (47) and (48) it is easily verified that the relation

$$
A=H(I+S)
$$

is valid, which is equivalent to (46). Since (34) holds we may therefore apply Theorem 8.1 in Chapter V of [5] to conclude that

$$
l_{\mathbb{C}}^{2}=\bigvee_{\mathrm{k}=1}^{+\infty} R_{\varkappa_{\mathrm{k}}}(A+P) .
$$

Provided we impose an additional restriction on the sequence $\left(\lambda_{\mathrm{m}}\right)_{\mathrm{m} \in \mathbb{N}^{+}}$, whose rôle is to control the gap between any two successive elements, we now proceed by showing that the set of all $\hat{p}_{\mathrm{k}}$ actually constitute a basis of $l_{\mathbb{C}}^{2}$. In a Hilbert space setting this means that there exists a unique sequence $\left(\hat{\mathrm{q}}_{\mathrm{k}}\right)_{\mathrm{k} \in \mathbb{N}^{+}}$ biorthogonal to $\left(\hat{\mathrm{p}}_{\mathrm{k}}\right)_{\mathrm{k} \in \mathbb{N}^{+}}$such that every $\mathrm{p} \in l_{\mathbb{C}}^{2}$ may be expanded in a unique way as the norm-convergent series

$$
\mathrm{p}=\sum_{\mathrm{k}=1}^{+\infty}\left(\mathrm{p}, \hat{\mathrm{q}}_{\mathrm{k}}\right)_{2} \hat{\mathrm{p}}_{\mathrm{k}}
$$

(see, e.g., Chapter VI in [5]). This, in turn, will lead to the following spectral result:

Main Theorem. With $\alpha \in(1,3)$ and $\theta \in\left(0, \frac{3-\alpha}{2} \beta\right)$, let us assume that

$$
\lambda_{\mathrm{m}+1}-\lambda_{\mathrm{m}} \geqslant c \exp \left[-\theta \lambda_{\mathrm{m}}\right]
$$


for every $\mathrm{m} \in \mathbb{N}^{+}$and some $c>0$ independent of $\mathrm{m}$. Then the set $\left(\hat{\mathrm{p}}_{\mathrm{k}}\right)_{\mathrm{k} \in \mathbb{N}^{+}}$of eigenvectors given by (19) provides a basis for $l_{\mathbb{C}}^{2}$ in the sense of (49). Moreover, for each $\mathrm{p} \in l_{\mathbb{C}}^{2}$ and every $\tau \in[0,+\infty)$ we have the norm-convergent spectral decomposition

$$
\exp [\tau A] \mathrm{p}=\sum_{\mathrm{k}=1}^{+\infty}\left(\mathrm{p}, \hat{\mathrm{q}}_{\mathrm{k}}\right)_{2} \exp \left[\tau \nu_{\mathrm{k}}\right] \hat{\mathrm{p}}_{\mathrm{k}}
$$

of the semigroup $\exp [\tau A]_{\tau \in[0,+\infty)}$ generated by $A$.

The proof of this theorem will be somewhat indirect and rests upon several preparatory results. We begin with the description of the biorthogonal sequence we alluded to above, and refer the reader to Appendix B for an alternative construction:

Proposition 4. If $\alpha \in(1,3)$, there exists a unique sequence $\left(\hat{\mathrm{q}}_{\mathrm{k}}\right)_{\mathrm{k} \in \mathbb{N}^{+}}$ biorthogonal to $\left(\hat{\mathrm{p}}_{\mathrm{k}}\right)_{\mathrm{k} \in \mathbb{N}^{+}}$.

Proof. Let us consider the orthogonal projection

$$
Q: l_{\mathbb{C}}^{2} \mapsto\left(\bigvee_{\mathrm{k}=2}^{+\infty} E_{\nu_{\mathrm{k}}}(A)\right)^{\perp} .
$$

We then have $\left\|Q \hat{\mathrm{p}}_{1}\right\|_{2}^{2}=\left(\hat{\mathrm{p}}_{1}, Q \hat{\mathrm{p}}_{1}\right)_{2} \neq 0$ as a consequence of a general fact proved in Appendix B, so that we may define

$$
\hat{\mathrm{q}}_{1}:=\left\|Q \hat{\mathrm{p}}_{1}\right\|_{2}^{-2} Q \hat{\mathrm{p}}_{1} .
$$

Consequently we get

$$
\left(\hat{\mathrm{p}}_{\mathrm{j}}, \hat{\mathrm{q}}_{1}\right)_{2}=\delta_{\mathrm{j}, 1}
$$

for every $\mathrm{j} \in \mathbb{N}^{+}$. Furthermore, since $A$ and $A^{*}$ are compact and the $\nu_{\mathrm{k}}$ are real, the eigenvalue equations for them when $\nu_{\mathrm{k}} \neq 0$ read $\left(A-\nu_{\mathrm{k}}\right) \hat{\mathrm{p}}_{\mathrm{k}}=0$ and $\left(A^{*}-\nu_{\mathrm{k}}\right) \hat{\mathrm{q}}_{\mathrm{k}}=0$ respectively, with $\hat{\mathrm{q}}_{\mathrm{k}} \in l_{\mathbb{C}}^{2}$ given by (26) in the second case. Therefore we have

$$
\left(\nu_{\mathrm{j}}-\nu_{\mathrm{k}}\right)\left(\hat{\mathrm{p}}_{\mathrm{j}}, \hat{\mathrm{q}}_{\mathrm{k}}\right)_{2}=\left(A \hat{\mathrm{p}}_{\mathrm{j}}, \hat{\mathrm{q}}_{\mathrm{k}}\right)_{2}-\left(\hat{\mathrm{p}}_{\mathrm{j}}, \mathrm{A}^{*} \hat{\mathrm{q}}_{\mathrm{k}}\right)_{2}=0,
$$

so that if $\mathrm{j}, \mathrm{k} \geqslant 2$ with $\mathrm{j} \neq \mathrm{k}$ then $\nu_{\mathrm{j}} \neq \nu_{\mathrm{k}}$ and so

$$
\left(\hat{\mathrm{p}}_{\mathrm{j}}, \hat{\mathrm{q}}_{\mathrm{k}}\right)_{2}=0 \text {. }
$$

A similar argument shows that

$$
\left(\hat{\mathrm{p}}_{1}, \hat{\mathrm{q}}_{\mathrm{k}}\right)_{2}=0
$$

for $k \geqslant 2$ and moreover we can impose $\left(\hat{p}_{k}, \hat{q}_{k}\right)_{2}=1$ by normalizing the eigenvectors accordingly, remembering that we may not have $\left(\hat{\mathrm{p}}_{\mathrm{k}}, \hat{\mathrm{q}}_{\mathrm{k}}\right)_{2}=0$ since this and (53) for $\mathrm{j} \neq \mathrm{k}$ would imply $\hat{\mathrm{q}}_{\mathrm{k}}=0$ by virtue of (45). Altogether we have

$$
\left(\hat{\mathrm{p}}_{\mathrm{j}}, \hat{\mathrm{q}}_{\mathrm{k}}\right)_{2}=\delta_{\mathrm{j}, \mathrm{k}}
$$


for all $j, k \in \mathbb{N}^{+}$as required, and the uniqueness of such a sequence is an immediate consequence of the completeness of the $\hat{p}_{k}$ guaranteed by Theorem 2 .

Our next step consists in renormalizing the $\hat{p}_{k}$ by defining the $\hat{r}_{k}$ as

$$
\hat{r}_{k}:=\left\{\begin{array}{cl}
\hat{p}_{1} & \text { for } k=1, \\
\hat{p}_{k}-\hat{p}_{1}, & \text { for } k \in\{2,3, \ldots\},
\end{array}\right.
$$

and in proving that the sequence $\left(\hat{r}_{\mathrm{k}}\right)_{\mathrm{k} \in \mathbb{N}^{+}}$constitutes a basis of $l_{\mathbb{C}}^{2}$. It first follows from (18) and (19) that

$$
\hat{r}_{\mathrm{k}, \mathrm{m}}=-\frac{\nu_{\mathrm{k}} \exp \left[-\beta \lambda_{\mathrm{m}}\right]}{Z_{\frac{\alpha+1}{2} \beta}\left(\nu_{\mathrm{k}}+b_{\mathrm{m}}\right)}
$$

for each $\mathrm{k} \in\{2,3, \ldots\}$ and every $\mathrm{m} \in \mathbb{N}^{+}$, and from the proof of Proposition 4 that the unique sequence $\left(\hat{\mathrm{s}}_{\mathrm{k}}\right)_{\mathrm{k} \in \mathbb{N}^{+}}$biorthogonal to $\left(\hat{r}_{\mathrm{k}}\right)_{\mathrm{k} \in \mathbb{N}^{+}}$is given by

$$
\hat{s}_{k}=\left\{\begin{array}{c}
\left\|\tilde{Q} \hat{p}_{1}\right\|_{2}^{-2} \tilde{Q} \hat{p}_{1} \quad \text { for } k=1, \\
\hat{q}_{k} \text { for } \mathrm{k} \in\{2,3, \ldots\},
\end{array}\right.
$$

where

$$
\tilde{Q}: l_{\mathbb{C}}^{2} \mapsto\left(\operatorname{cl} \operatorname{span}\left\{\hat{r}_{\mathrm{k}}, \mathrm{k} \in\{2,3, \ldots\}\right\}\right)^{\perp}
$$

denotes the orthogonal projection onto the indicated space, with cl span referring to the closure of the span in $l_{\mathbb{C}}^{2}$. In order to prove that the $\hat{r}_{\mathrm{k}}$ provide a basis for $l_{\mathbb{C}}^{2}$ we first need some estimates related to the localization properties of the $\nu_{\mathrm{k}}$. We begin with the following:

Lemma 1. Under the sole conditions $\alpha>1, \beta>0$, there exists a constant $c_{\alpha, \beta}>0$ depending on $\alpha$ and $\beta$ such that the estimate

$$
\mathrm{b}_{\mathrm{m}}-\mathrm{b}_{\mathrm{m}+1} \geqslant c_{\alpha, \beta} \exp \left[-\left(\frac{\alpha-1}{2} \beta+\theta\right) \lambda_{\mathrm{m}}\right]
$$

holds for each $\mathrm{m} \in \mathbb{N}^{+}$and every $\theta>0$, where $\mathrm{b}_{\mathrm{m}}$ is given by (18).

Proof. From (18) we first have

$$
\begin{aligned}
& \mathrm{b}_{\mathrm{m}}-\mathrm{b}_{\mathrm{m}+1} \\
= & Z_{\frac{\alpha+1}{2} \beta} \exp \left[-\frac{\alpha-1}{2} \beta \lambda_{\mathrm{m}}\right]\left(1-\exp \left[-\frac{\alpha-1}{2} \beta\left(\lambda_{\mathrm{m}+1}-\lambda_{\mathrm{m}}\right)\right]\right) \\
\geqslant & Z_{\frac{\alpha+1}{2} \beta} \exp \left[-\frac{\alpha-1}{2} \beta \lambda_{\mathrm{m}}\right]\left(1+\frac{2}{(\alpha-1) \beta}\left(\lambda_{\mathrm{m}+1}-\lambda_{\mathrm{m}}\right)^{-1}\right)^{-1}
\end{aligned}
$$


as a consequence of the elementary inequality

$$
1-\exp [-x] \geqslant\left(1+x^{-1}\right)^{-1}
$$

valid for every $x>0$. Furthermore, from (50) we get the lower bounds

$$
\begin{aligned}
& \left(1+\frac{2}{(\alpha-1) \beta}\left(\lambda_{\mathrm{m}+1}-\lambda_{\mathrm{m}}\right)^{-1}\right)^{-1} \\
\geqslant & \left(1+\frac{2}{c(\alpha-1) \beta} \exp \left[\theta \lambda_{\mathrm{m}}\right]\right)^{-1}=\exp \left[-\theta \lambda_{\mathrm{m}}\right]\left(\exp \left[-\theta \lambda_{\mathrm{m}}\right]+\frac{2}{c(\alpha-1) \beta}\right)^{-1} \\
\geqslant & \exp \left[-\theta \lambda_{\mathrm{m}}\right]\left(1+\frac{2}{c(\alpha-1) \beta}\right)^{-1}
\end{aligned}
$$

for the third factor on the right-hand side of the inequality in (59) since $\theta>0$, and since we may assume $\lambda_{\mathrm{m}}>0$ for each $\mathrm{m}$ without restricting the generality. The substitution of the last estimate into (59) then leads to (58) with an obvious choice for $c_{\alpha, \beta}$.

Next we have:

Lemma 2. Under the sole conditions $\alpha>1, \beta>0$, there exists a constant $\hat{c}_{\alpha, \beta}>0$ depending on $\alpha$ and $\beta$ such that the inequality

$$
\left|\nu_{\mathrm{k}}+\mathrm{b}_{\mathrm{m}}\right| \geqslant c_{\alpha, \beta} \exp \left[-\left(\frac{\alpha-1}{2} \beta+\theta\right) \lambda_{\mathrm{m}}\right]
$$

holds for all sufficiently large $\mathrm{k}$, each $\mathrm{m} \neq \mathrm{k}$ and every $0<\theta<\beta$. Moreover, for all sufficiently large $\mathrm{k}$ and $\mathrm{m}=\mathrm{k}$ we have

$$
\left|\nu_{\mathrm{k}}+\mathrm{b}_{\mathrm{k}}\right| \leq \hat{c}_{\alpha, \beta, \lambda_{1}} \exp \left[-\frac{\alpha+1}{2} \beta \lambda_{\mathrm{k}}\right]
$$

for some suitable $\hat{c}_{\alpha, \beta, \lambda_{1}}>0$.

Proof. Let us first consider the case $\mathrm{m}>\mathrm{k} \in\{2,3, \ldots\}$. From the localization property of the eigenvalues stated in (c) of Theorem 1 we then have

$$
b_{m}-b_{k-1}<\nu_{k}+b_{m}<b_{m}-b_{k}<0
$$

and thereby

$$
\left|\nu_{k}+b_{m}\right| \geqslant b_{k}-b_{m} \geqslant b_{m-1}-b_{m}
$$

since $\mathrm{m}-1 \geqslant \mathrm{k}$. Therefore, applying (59) with $\mathrm{m}-1$ instead of $\mathrm{m}$ and using the fact that $\lambda_{\mathrm{m}}>\lambda_{\mathrm{m}-1}$ we obtain

$$
\left|\nu_{\mathrm{k}}+\mathrm{b}_{\mathrm{m}}\right| \geqslant c_{\alpha, \beta} \exp \left[-\left(\frac{\alpha-1}{2} \beta+\theta\right) \lambda_{\mathrm{m}-1}\right] \geqslant c_{\alpha, \beta} \exp \left[-\left(\frac{\alpha-1}{2} \beta+\theta\right) \lambda_{\mathrm{m}}\right]
$$


which is (60) for this case.

Let us now assume that $m<k-1$. From (62) we have this time

$$
\left|\nu_{k}+b_{m}\right| \geqslant b_{m}-b_{k-1} \geqslant b_{m}-b_{m+1}
$$

since $m+1 \leqslant k-1$, so that (60) again follows directly from (59).

The remaining cases are a bit trickier. Thus, let us take $m=k$ and observe that (27) along with the localization property of the eigenvalues imply the identity

$$
\frac{\exp \left[-\frac{\alpha+1}{2} \beta \lambda_{\mathrm{k}}\right]}{\left|\nu_{\mathrm{k}}+b_{\mathrm{k}}\right|}=\sum_{\mathrm{m}=1}^{\mathrm{k}-1} \frac{\exp \left[-\frac{\alpha+1}{2} \beta \lambda_{\mathrm{m}}\right]}{\left|\nu_{\mathrm{k}}+b_{\mathrm{m}}\right|}-\sum_{\mathrm{m}=\mathrm{k}+1}^{+\infty} \frac{\exp \left[-\frac{\alpha+1}{2} \beta \lambda_{\mathrm{m}}\right]}{\left|\nu_{\mathrm{k}}+b_{\mathrm{m}}\right|}
$$

for every $k \in\{2,3, \ldots\}$. We then proceed by getting a lower bound for each term on the right-hand side of (64). On the one hand we have

$$
\sum_{\mathrm{m}=1}^{\mathrm{k}-1} \frac{\exp \left[-\frac{\alpha+1}{2} \beta \lambda_{\mathrm{m}}\right]}{\left|\nu_{\mathrm{k}}+b_{\mathrm{m}}\right|} \geqslant \frac{\exp \left[-\frac{\alpha+1}{2} \beta \lambda_{1}\right]}{\left|\nu_{\mathrm{k}}+b_{1}\right|} \geqslant \frac{\exp \left[-\frac{\alpha+1}{2} \beta \lambda_{1}\right]}{b_{1}}=\frac{\exp \left[-\beta \lambda_{1}\right]}{Z_{\frac{\alpha+1}{2} \beta}}
$$

as a consequence of (18) since $\left|\nu_{\mathrm{k}}+b_{1}\right| \leqslant b_{1}$. On the other hand we obtain

$$
\sum_{\mathrm{m}=\mathrm{k}+1}^{+\infty} \frac{\exp \left[-\frac{\alpha+1}{2} \beta \lambda_{\mathrm{m}}\right]}{\left|\nu_{\mathrm{k}}+b_{\mathrm{m}}\right|} \leqslant \frac{1}{c_{\alpha, \beta}} \sum_{\mathrm{m}=\mathrm{k}+1}^{+\infty} \exp \left[-(\beta-\theta) \lambda_{\mathrm{m}}\right]
$$

from (63), so that the substitution of (65) and (66) into (64) leads to

$$
\frac{\exp \left[-\frac{\alpha+1}{2} \beta \lambda_{\mathrm{k}}\right]}{\left|\nu_{\mathrm{k}}+b_{\mathrm{k}}\right|} \geqslant \frac{\exp \left[-\beta \lambda_{1}\right]}{Z_{\frac{\alpha+1}{2} \beta}}-\frac{1}{c_{\alpha, \beta}} \sum_{\mathrm{m}=\mathrm{k}+1}^{+\infty} \exp \left[-(\beta-\theta) \lambda_{\mathrm{m}}\right] .
$$

Since $\theta<\beta$, the second term on the right-hand side of the preceding expression tends to zero as $k$ becomes large by virtue of (11). In particular, there exists $\mathrm{k}^{*}$ such that for every $k \geqslant k^{*}$ we have

$$
\sum_{\mathrm{m}=\mathrm{k}+1}^{+\infty} \exp \left[-(\beta-\theta) \lambda_{\mathrm{m}}\right] \leqslant \frac{c_{\alpha, \beta}}{2 Z_{\frac{\alpha+1}{2} \beta}} \exp \left[-\beta \lambda_{1}\right]
$$

which gives

$$
\frac{\exp \left[-\frac{\alpha+1}{2} \beta \lambda_{\mathrm{k}}\right]}{\left|\nu_{\mathrm{k}}+b_{\mathrm{k}}\right|} \geqslant \frac{\exp \left[-\beta \lambda_{1}\right]}{2 Z_{\frac{\alpha+1}{2} \beta}}
$$

according to (67), and thereby (61) with an obvious choice for $\hat{c}_{\alpha, \beta, \lambda_{1}}$.

It remains to consider the case $\mathrm{m}=\mathrm{k}-1$. Using once again the localization property we have

$$
\left|\nu_{\mathrm{k}}+b_{\mathrm{k}-1}\right|=\mathrm{b}_{\mathrm{k}-1}-\mathrm{b}_{\mathrm{k}}-\left|\nu_{\mathrm{k}}+b_{\mathrm{k}}\right|
$$


and therefore the estimate

$$
\begin{aligned}
\left|\nu_{\mathrm{k}}+b_{\mathrm{k}-1}\right| & \geqq c_{\alpha, \beta} \exp \left[-\left(\frac{\alpha-1}{2} \beta+\theta\right) \lambda_{\mathrm{k}-1}\right]-\hat{c} \exp \left[-\frac{\alpha+1}{2} \beta \lambda_{\mathrm{k}-1}\right](68) \\
& =\exp \left[-\left(\frac{\alpha-1}{2} \beta+\theta\right) \lambda_{\mathrm{k}-1}\right]\left\{c_{\alpha, \beta}-\hat{c} \exp \left[-(\beta-\theta) \lambda_{\mathrm{k}-1}\right]\right\}
\end{aligned}
$$

for every $k \geqslant k^{*}$ as a consequence of Lemma 1 with $m=k-1$ and (61). Now since $\theta<\beta$ and $\lambda_{\mathrm{k}} \rightarrow+\infty$ as $\mathrm{k} \rightarrow+\infty$, there exists $\mathrm{k}^{* *}$ such that for every $\mathrm{k} \geqslant \mathrm{k}^{* *}$ we have

$$
\hat{c} \exp \left[-(\beta-\theta) \lambda_{\mathrm{k}-1}\right] \leqslant \frac{c_{\alpha, \beta}}{2} .
$$

Consequently, the substitution of the preceding relation into (68) gives

$$
\left|\nu_{\mathrm{k}}+b_{\mathrm{k}-1}\right| \geqslant \frac{c_{\alpha, \beta}}{2} \exp \left[-\left(\frac{\alpha-1}{2} \beta+\theta\right) \lambda_{\mathrm{k}-1}\right]
$$

for all sufficiently large $\mathrm{k}$.

The critical result is now the following:

Proposition 5. Let us assume that the same hypotheses as in the main theorem hold. Then for every $\mathrm{p} \in l_{\mathbb{C}}^{2}$ we have the norm-convergent series expansion

$$
\mathrm{p}=\sum_{\mathrm{k}=1}^{+\infty}\left(\mathrm{p},\left\|\hat{\mathrm{r}}_{\mathrm{k}}\right\|_{2} \hat{\mathrm{s}}_{\mathrm{k}}\right)_{2} \frac{\hat{\mathrm{r}}_{\mathrm{k}}}{\left\|\hat{\mathrm{r}}_{\mathrm{k}}\right\|_{2}}
$$

where $\hat{\mathrm{r}}_{\mathrm{k}}$ and $\hat{\mathrm{s}}_{\mathrm{k}}$ are given by (55) and (57), respectively. Equivalently, the sequence $\left(\hat{\mathrm{r}}_{\mathrm{k}}\right)_{\mathrm{k} \in \mathbb{N}^{+}}$provides a basis for $l_{\mathbb{C}^{2}}^{2}$.

Proof. It is plain that the $\hat{r}_{\mathrm{k}}$ form a complete set in $l_{\mathbb{C}}^{2}$ since the $\hat{p}_{\mathrm{k}}$ do. Next, we prove that

$$
\sum_{j=1}^{+\infty} \sum_{k=1, k \neq j}^{+\infty}\left|\left(\frac{\hat{r}_{j}}{\left\|\hat{r}_{j}\right\|_{2}}, \frac{\hat{r}_{k}}{\left\|\hat{r}_{k}\right\|_{2}}\right)_{2}\right|^{2}<+\infty
$$

by using the inequalities we provided in Lemma 2. For the inner product in the preceding expression we first have

$$
\left(\frac{\hat{r}_{\mathrm{j}}}{\left\|\hat{r}_{\mathrm{j}}\right\|_{2}}, \frac{\hat{\mathrm{r}}_{\mathrm{k}}}{\left\|\hat{r}_{\mathrm{k}}\right\|_{2}}\right)_{2}=\frac{1}{\left\|\hat{r}_{\mathrm{j}}\right\|_{2}\left\|\hat{r}_{\mathrm{k}}\right\|_{2}}\left(\hat{\mathrm{r}}_{\mathrm{j}, \mathrm{j}} \hat{r}_{\mathrm{k}, \mathrm{j}}+\hat{\mathrm{r}}_{\mathrm{j}, \mathrm{k}} \hat{\mathrm{r}}_{\mathrm{k}, \mathrm{k}}+\sum_{\mathrm{m}=1, \mathrm{~m} \neq \mathrm{j}, \mathrm{k}}^{+\infty} \hat{\mathrm{r}}_{\mathrm{j}, \mathrm{m}} \hat{r}_{\mathrm{k}, \mathrm{m}}\right)
$$

and therefore the estimate

$$
\left|\left(\frac{\hat{r}_{j}}{\left\|\hat{r}_{j}\right\|_{2}}, \frac{\hat{r}_{k}}{\left\|\hat{r}_{k}\right\|_{2}}\right)_{2}\right| \leqslant \frac{\left|\hat{r}_{k, j}\right|}{\left|\hat{r}_{k, k}\right|}+\frac{\left|\hat{r}_{j, k}\right|}{\left|\hat{r}_{j, j}\right|}+\sum_{m=1, m \neq j, k}^{+\infty} \frac{\left|\hat{r}_{j, m}\right|\left|\hat{r}_{k, m}\right|}{\left|\hat{r}_{j, j}\right|\left|\hat{r}_{k, k}\right|}
$$


by virtue of the inequalities $\left\|\hat{r}_{\mathrm{j}}\right\|_{2} \geqslant\left|\hat{\mathrm{r}}_{\mathrm{j}, \mathrm{j}}\right|$ and $\left\|\hat{\mathrm{r}}_{\mathrm{k}}\right\|_{2} \geqslant\left|\hat{\mathrm{r}}_{\mathrm{k}, \mathrm{k}}\right|$. Now from (56) we have

$$
\frac{\left|\hat{r}_{\mathrm{k}, \mathrm{j}}\right|}{\left|\hat{\mathrm{r}}_{\mathrm{k}, \mathrm{k}}\right|}=\frac{\left|\nu_{\mathrm{k}}+b_{\mathrm{k}}\right|}{\left|\nu_{\mathrm{k}}+b_{\mathrm{j}}\right|} \exp \left[-\beta\left(\lambda_{\mathrm{j}}-\lambda_{\mathrm{k}}\right)\right]
$$

and

$$
\frac{\left|\hat{r}_{\mathrm{j}, \mathrm{k}}\right|}{\left|\hat{\mathrm{r}}_{\mathrm{j}, \mathrm{j}}\right|}=\frac{\left|\nu_{\mathrm{j}}+b_{\mathrm{j}}\right|}{\left|\nu_{\mathrm{j}}+b_{\mathrm{k}}\right|} \exp \left[-\beta\left(\lambda_{\mathrm{k}}-\lambda_{\mathrm{j}}\right)\right]
$$

for all $\mathrm{j}$ and $\mathrm{k}$, so that by means of inequalities (60) and (61) we obtain

$$
\frac{\left|\hat{\mathrm{r}}_{\mathrm{k}, \mathrm{j}}\right|}{\left|\hat{\mathrm{r}}_{\mathrm{k}, \mathrm{k}}\right|} \leqslant \frac{\hat{c}}{c_{\alpha, \beta}} \exp \left[-\left(\frac{3-\alpha}{2} \beta-\theta\right) \lambda_{\mathrm{j}}\right] \exp \left[-\frac{\alpha-1}{2} \beta \lambda_{\mathrm{k}}\right]
$$

for all sufficiently large $k$ and every $\mathrm{j} \neq \mathrm{k}$, and similarly

$$
\frac{\left|\hat{\mathrm{r}}_{\mathrm{j}, \mathrm{k}}\right|}{\left|\hat{\mathrm{r}}_{\mathrm{j}, \mathrm{j}}\right|} \leqslant \frac{\hat{c}}{c_{\alpha, \beta}} \exp \left[-\left(\frac{3-\alpha}{2} \beta-\theta\right) \lambda_{\mathrm{k}}\right] \exp \left[-\frac{\alpha-1}{2} \beta \lambda_{\mathrm{j}}\right]
$$

for all sufficiently large $\mathrm{j}$ and every $\mathrm{k} \neq \mathrm{j}$. By the same token we have

$$
\begin{aligned}
& \sum_{\mathrm{m}=1, \mathrm{~m} \neq \mathrm{j}, \mathrm{k}}^{+\infty} \frac{\left|\hat{\mathrm{r}}_{\mathrm{j}, \mathrm{m}}\right|\left|\hat{\mathrm{r}}_{\mathrm{k}, \mathrm{m}}\right|}{\left|\hat{\mathrm{r}}_{\mathrm{j}, \mathrm{j}}\right| \hat{\mathrm{r}}_{\mathrm{k}, \mathrm{k}} \mid} \\
\leqslant & \left(\frac{\hat{c}}{c_{\alpha, \beta}}\right)^{2} \sum_{\mathrm{m}=1, \mathrm{~m} \neq \mathrm{j}, \mathrm{k}}^{+\infty} \exp \left[-((3-\alpha) \beta-2 \theta) \lambda_{\mathrm{m}}\right] \exp \left[-\frac{\alpha-1}{2} \beta\left(\lambda_{\mathrm{j}}+\lambda_{\mathrm{k}}\right)\right] \\
\leqslant & \left(\frac{\hat{c}}{c_{\alpha, \beta}}\right)^{2} Z_{(3-\alpha) \beta-2 \theta} \exp \left[-\frac{\alpha-1}{2} \beta\left(\lambda_{\mathrm{j}}+\lambda_{\mathrm{k}}\right)\right]
\end{aligned}
$$

by virtue of (1) as a consequence of the hypotheses regarding $\alpha$ and $\theta$, for all sufficiently large $\mathrm{j}$ and $\mathrm{k}$ with $\mathrm{j} \neq \mathrm{k}$. Let us now define

$$
\kappa:=\left(\frac{3-\alpha}{2} \beta-\theta\right) \wedge \frac{\alpha-1}{2} \beta,
$$

the smaller of the two numbers, which is positive. Then, by using estimates (71)-(74) we get

$$
\left|\left(\frac{\hat{\mathrm{r}}_{\mathrm{j}}}{\left\|\hat{\mathrm{r}}_{\mathrm{j}}\right\|_{2}}, \frac{\hat{\mathrm{r}}_{\mathrm{k}}}{\left\|\hat{\mathrm{r}}_{\mathrm{k}}\right\|_{2}}\right)_{2}\right| \leqslant\left(\frac{2 \hat{c}}{c_{\alpha, \beta}}+\left(\frac{\hat{c}}{c_{\alpha, \beta}}\right)^{2} Z_{(3-\alpha) \beta-2 \theta}\right) \exp \left[-\kappa\left(\lambda_{\mathrm{j}}+\lambda_{\mathrm{k}}\right)\right]
$$

and there exist $\mathrm{J}, \mathrm{K} \in \mathbb{N}^{+}$such that

$$
\begin{aligned}
& \sum_{j=J}^{+\infty} \sum_{k=K, k \neq j}^{+\infty}\left|\left(\frac{\hat{r}_{j}}{\left\|\hat{r}_{j}\right\|_{2}}, \frac{\hat{r}_{k}}{\left\|\hat{r}_{k}\right\|_{2}}\right)_{2}\right|^{2} \\
\leqslant & c_{\alpha, \beta, \theta} \sum_{j=J}^{+\infty} \sum_{k=K, k \neq j}^{+\infty} \exp \left[-2 \kappa\left(\lambda_{\mathrm{j}}+\lambda_{\mathrm{k}}\right)\right] \leqslant c_{\alpha, \beta, \theta} Z_{2 \kappa}^{2}<\infty
\end{aligned}
$$


for some $c_{\alpha, \beta, \theta}$ because of (11), which proves (70) and thus guarantees the existence of a large enough $\mathrm{N}^{*} \in \mathbb{N}^{+}$such that

$$
\sum_{j, k \geqslant N^{*}, j \neq k}^{+\infty}\left|\left(\frac{\hat{r}_{j}}{\left\|\hat{r}_{j}\right\|_{2}}, \frac{\hat{r}_{k}}{\left\|\hat{r}_{k}\right\|_{2}}\right)_{2}\right|^{2}<1 .
$$

Let us now consider the subspace of $l_{\mathbb{C}}^{2}$ defined by

$$
h_{\mathrm{N}^{*}}:=\operatorname{cl} \operatorname{span}\left\{\hat{\mathrm{r}}_{\mathrm{k}}, \mathrm{k} \in\left\{\mathrm{N}^{*}, \mathrm{~N}^{*}+1, \ldots\right\}\right\}
$$

where $\mathrm{N}^{*}$ is as in (75). Let $M_{\hat{\mathrm{r}}, \mathrm{N}^{*}}$ stand for the infinite Gram matrix of the normalized $\hat{r}_{\mathrm{k}}$ with $\mathrm{k}$ as in (76) and $\mathbb{I}_{\mathrm{N}^{*}}$ for the identity operator on $h_{\mathrm{N}^{*}}$. We then have

$$
\left(\left(M_{\hat{\mathrm{r}}, \mathrm{N}^{*}}-\mathbb{I}_{\mathrm{N}^{*}}\right) \mathrm{p}_{\mathrm{N}^{*}}\right)_{\mathrm{j}}=\sum_{\mathrm{k}=\mathrm{N}^{*}, \mathrm{k} \neq \mathrm{j}}^{+\infty}\left(\frac{\hat{\mathrm{r}}_{\mathrm{j}}}{\left\|\hat{\mathrm{r}}_{\mathrm{j}}\right\|_{2}}, \frac{\hat{\mathrm{r}}_{\mathrm{k}}}{\left\|\hat{\mathrm{r}}_{\mathrm{k}}\right\|_{2}}\right)_{2} p_{\mathrm{N}^{*}, \mathrm{k}}
$$

where $\mathrm{j} \in \mathbb{N}^{+}$with $\mathrm{j} \geqslant \mathrm{N}^{*}$ and $\mathrm{p}_{\mathrm{N}^{*}} \in h_{\mathrm{N}^{*}}$. In this manner it follows from (75) that the transformation $M_{\hat{\mathrm{r}}, \mathrm{N}^{*}}-\mathbb{I}_{\mathrm{N}^{*}}$ is a Hilbert-Schmidt operator on $h_{\mathrm{N}^{*}}$ with

$$
\left\|M_{\hat{\mathrm{r}}, \mathrm{N}^{*}}-\mathbb{I}_{\mathrm{N}^{*}}\right\|_{\mathrm{HS}, \mathrm{N}^{*}}^{2}=\sum_{\mathrm{j}, \mathrm{k} \geqslant \mathrm{N}^{*}, \mathrm{j} \neq \mathrm{k}}^{+\infty}\left|\left(\frac{\hat{\mathrm{r}}_{\mathrm{j}}}{\left\|\hat{\mathrm{r}}_{\mathrm{j}}\right\|_{2}}, \frac{\hat{\mathrm{r}}_{\mathrm{k}}}{\left\|\hat{\mathrm{r}}_{\mathrm{k}}\right\|_{2}}\right)_{2}\right|^{2}<1,
$$

where $\|\cdot\|_{\mathrm{HS}, \mathrm{N}^{*}}$ stands for the Hilbert-Schmidt norm there. Therefore, we have a fortiori

$$
\left\|M_{\hat{r}, \mathrm{~N}^{*}}-\mathbb{I}_{\mathrm{N}^{*}}\right\|_{\infty, \mathrm{N}^{*}}<1
$$

where $\|\cdot\|_{\infty, \mathrm{N}^{*}}$ denotes the usual sup-norm of the linear bounded operators on $h_{\mathrm{N}^{*}}$. This proves that $M_{\hat{\mathrm{r}}, \mathrm{N}^{*}}=\mathbb{I}_{\mathrm{N}^{*}}-\left(\mathbb{I}_{\mathrm{N}^{*}}-M_{\hat{\mathrm{r}}, \mathrm{N}^{*}}\right)$ is such an operator whose inverse is also bounded and given by the corresponding Neumann series. Consequently, the $\hat{r}_{\mathrm{k}}$ with $\mathrm{k} \in\left\{\mathrm{N}^{*}, \mathrm{~N}^{*}+1, \ldots\right\}$ constitute a basis of $h_{\mathrm{N}^{*}}$ according to the fourth assertion of Theorem 2.1 in Chapter VI of [5], that is, for every $\mathrm{p}_{\mathrm{N}^{*}} \in$ $h_{\mathrm{N}^{*}}$ we have the norm-convergent series expansion

$$
\mathrm{p}_{\mathrm{N}^{*}}=\sum_{\mathrm{k}=\mathrm{N}^{*}}^{+\infty}\left(\mathrm{p}_{\mathrm{N}^{*}}, \hat{\mathrm{s}}_{\mathrm{k}}\right)_{2} \hat{\mathrm{r}}_{\mathrm{k}}
$$

In order to get the result we want it remains to prove that we can complete the basis just constructed with $\hat{r}_{1}, \ldots, \hat{r}_{\mathrm{N}^{*}-1}$. Let $\mathrm{V}_{\mathrm{N}^{*}}$ be the $\left(\mathrm{N}^{*}-1\right)$-dimensional subspace of $l_{\mathbb{C}}^{2}$ generated by these vectors. It follows from the definitions of $\mathrm{V}_{\mathrm{N}^{*}}$, $h_{\mathrm{N}^{*}}$ and from the completeness of all the $\hat{\mathrm{r}}_{\mathrm{k}}$ that

$$
l_{\mathbb{C}}^{2}=\mathrm{V}_{\mathrm{N}^{*}} \oplus h_{\mathrm{N}^{*}}
$$

as an algebraic direct sum. Therefore, any $\mathrm{p} \in l_{\mathbb{C}}^{2}$ may be written as

$$
\mathrm{p}=\sum_{\mathrm{k}=1}^{\mathrm{N}^{*}-1} \gamma_{\mathrm{k}} \hat{\mathrm{r}}_{\mathrm{k}}+\sum_{\mathrm{k}=\mathrm{N}^{*}}^{+\infty}\left(\mathrm{p}_{\mathrm{N}^{*}}, \hat{\mathrm{s}}_{\mathrm{k}}\right)_{2} \hat{\mathrm{r}}_{\mathrm{k}}
$$


with some $\gamma_{\mathrm{k}} \in \mathbb{C}$ and $\mathrm{p}_{\mathrm{N}^{*}} \in h_{\mathrm{N}^{*}}$. Consequently, using the biorthogonality properties

$$
\left(\hat{\mathrm{r}}_{\mathrm{j}}, \hat{\mathrm{s}}_{\mathrm{k}}\right)_{2}=\delta_{\mathrm{j}, \mathrm{k}}
$$

stemming from (55) and (57) we get

$$
\left(\mathrm{p}, \hat{\mathrm{s}}_{\mathrm{k}}\right)_{2}=\left\{\begin{array}{c}
\gamma_{\mathrm{k}} \text { for } \mathrm{k} \in\left\{1, \ldots, \mathrm{N}^{*}-1\right\}, \\
\left(\mathrm{p}_{\mathrm{N}^{*}}, \hat{\mathrm{s}}_{\mathrm{k}}\right)_{2} \quad \text { for } \mathrm{k} \in\left\{\mathrm{N}^{*}, \mathrm{~N}^{*}+1, \ldots\right\}
\end{array}\right.
$$

and thereby

$$
p=\sum_{k=1}^{N^{*}-1}\left(p, \hat{s}_{k}\right)_{2} \hat{r}_{k}+\sum_{k=N^{*}}^{+\infty}\left(p_{N^{*}}, \hat{s}_{k}\right)_{2} \hat{r}_{k}=\sum_{k=1}^{+\infty}\left(p, \hat{s}_{k}\right)_{2} \hat{r}_{k},
$$

as desired.

Remark. According to the theorem we just referred to in the above proof, the basis $\left(\hat{r}_{\mathrm{k}}\right)$ of $h_{\mathrm{N}^{*}}$ with $\mathrm{k} \in\left\{\mathrm{N}^{*}, \mathrm{~N}^{*}+1, \ldots\right\}$ is actually a Riesz basis, that is, one which may be obtained by a suitable deformation of an orthonormal basis involving bounded invertible transformations. That notion may be traced back to the original considerations set forth in Section XXXVII in Chapter VII of [10], whose abstract version appears in Section 86 in Chapter V of [11.

We are now ready for the following:

Proof of the main theorem. Owing to (55) and (57) we first have

$$
\sum_{k=2}^{N}\left(p, \hat{q}_{k}\right)_{2} \hat{p}_{k}=\sum_{k=1}^{N}\left(p, \hat{s}_{k}\right)_{2} \hat{r}_{k}+\left(\sum_{k=2}^{N}\left(p, \hat{q}_{k}\right)_{2}\right) \hat{p}_{1}-\left(p, \hat{s}_{1}\right)_{2} \hat{p}_{1}
$$

for each $\mathrm{p} \in l_{\mathbb{C}}^{2}$ and every $\mathrm{N} \in \mathbb{N}^{+}, \mathrm{N} \geqslant 2$. The issue being to establish (49) from (69), it is then necessary to prove the convergence of the middle term on the right-hand side of (77) as $N \rightarrow+\infty$ by an independent argument. For this it is sufficient to take the inner product of the preceding equality by $\hat{q}_{1}$ given by (52). In so doing we obtain

$$
\sum_{k=2}^{N}\left(p, \hat{q}_{k}\right)_{2}=\left(p, \hat{s}_{1}\right)_{2}-\left(\sum_{k=1}^{N}\left(p, \hat{s}_{k}\right)_{2} \hat{r}_{k}, \hat{q}_{1}\right)_{2}
$$

from the biorthogonality properties of Proposition 4, so that we have

$$
\sum_{\mathrm{k}=2}^{+\infty}\left(\mathrm{p}, \hat{\mathrm{q}}_{\mathrm{k}}\right)_{2}=\left(\mathrm{p}, \hat{\mathrm{s}}_{1}-\hat{\mathrm{q}}_{1}\right)_{2}
$$


since (69) implies the convergence of the second term on the right-hand side of (78) to $\left(\mathrm{p}, \hat{\mathrm{q}}_{1}\right)_{2}$. Therefore, letting $\mathrm{N} \rightarrow+\infty$ in (77) while using (79) we get

$$
\sum_{k=2}^{+\infty}\left(p, \hat{q}_{k}\right)_{2} \hat{p}_{k}=p+\left(p, \hat{s}_{1}-\hat{q}_{1}\right)_{2} \hat{p}_{1}-\left(p, \hat{s}_{1}\right)_{2} \hat{p}_{1}
$$

in the sense of norm-convergence for every $\mathrm{p} \in l_{\mathbb{C}}^{2}$, which is (49). Finally, (51) is a direct consequence of the continuity properties of the semigroup and of the spectral properties of $A$.

The spectral decomposition of the main theorem now leads to the desired description of the dynamics generated by (5) and of its ultimate behavior for large times, where we keep ordering the negative eigenvalues of $A$ as $\nu_{\mathrm{k}}<\nu_{\mathrm{k}+1}$ for every $k \in\{2,3, \ldots\}$ and where $\|\cdot\|_{\infty}$ stands for the sup-norm of the linear bounded operators on $l_{\mathbb{C}}^{2}$ :

Corollary. Let us assume that the same hypotheses as in the main theorem hold. Then the following statements are valid:

(a) Let $\mathrm{p}^{*}=\left(p_{\mathrm{m}}^{*}\right)_{\mathrm{m} \in \mathbb{N}^{+}}$be any initial condition satisfying (4). Then we have

$$
\left(\exp [\tau A] \mathrm{p}^{*}\right)_{\mathrm{m}} \geqslant 0, \quad \sum_{\mathrm{m}=1}^{+\infty}\left(\exp [\tau A] \mathrm{p}^{*}\right)_{\mathrm{m}}=1
$$

for every $\tau \in[0,+\infty)$.

(b) The Lyapunov exponent of the semigroup $\exp [\tau A]_{\tau \in[0,+\infty)}$ is given by

$$
\lim _{\tau \rightarrow+\infty} \frac{\ln \|\exp [\tau A]\|_{\infty}}{\tau}=0
$$

(c) For each $\mathrm{N} \in \mathbb{N}^{+}, \mathrm{N} \geqslant 2$, there exists a constant $c_{\mathrm{N}}>0$ such that for every $\mathrm{p}^{*} \in\left(\mathrm{\vee}_{\mathrm{k}=\mathrm{N}+1}^{+\infty} E_{\nu_{\mathrm{k}}}\left(A^{*}\right)\right)^{\perp}$ we have the exponential decay estimate

$$
\left\|\exp [\tau A] \mathrm{p}^{*}-\left(\mathrm{p}^{*}, \hat{\mathrm{q}}_{1}\right)_{2} \hat{\mathrm{p}}_{1}\right\|_{2} \leqslant c_{\mathrm{N}} \exp \left[-\tau\left|\nu_{\mathrm{N}}\right|\right]\left\|\mathrm{p}^{*}\right\|_{2} .
$$

In particular we have

$$
\left|\left(\exp [\tau A] \mathrm{p}^{*}\right)_{\mathrm{m}}-\left(\mathrm{p}^{*}, \hat{\mathrm{q}}_{1}\right)_{2} \hat{\mathrm{p}}_{1, \mathrm{~m}}\right| \leqslant c_{\mathrm{N}} \exp \left[\tau \nu_{\mathrm{N}}\right]\left\|\mathrm{p}^{*}\right\|_{2}
$$

for each $\mathrm{m} \in \mathbb{N}^{+}$and every $\tau \in(0,+\infty)$, where $\hat{\mathrm{q}}_{1}$ is given by (52).

Proof. The proof of Statement (a) follows immediately from (4), the continuity of $\tau \mapsto \exp [\tau A] \mathrm{p}^{*}$ and the summation on both sides of (15) over $\mathrm{m} \in \mathbb{N}^{+}$.

Statement (b) is a consequence of the very last part of Theorem 1 since

$$
\lim _{\tau \rightarrow+\infty} \frac{\ln \|\exp [\tau A]\|_{\infty}}{\tau}=\max _{\mathrm{k} \in\{1,2, \ldots .\}} \nu_{\mathrm{k}}
$$


as an application of Theorem 4.1 in Chapter I of [2].

As for Statement (c), with $\mathrm{p}^{*} \in\left(\mathrm{\vee}_{\mathrm{k}=\mathrm{N}+1}^{+\infty} E_{\nu_{\mathrm{k}}}\left(A^{*}\right)\right)^{\perp}$ we have

$$
\exp [\tau A] \mathrm{p}^{*}-\left(\mathrm{p}^{*}, \hat{\mathrm{q}}_{1}\right)_{2} \hat{\mathrm{p}}_{1}=\sum_{\mathrm{k}=2}^{\mathrm{N}}\left(\mathrm{p}^{*}, \hat{\mathrm{q}}_{\mathrm{k}}\right)_{2} \exp \left[\tau \nu_{\mathrm{k}}\right] \hat{\mathrm{p}}_{\mathrm{k}}
$$

from (51) since then $\left(\mathrm{p}^{*}, \hat{\mathrm{q}}_{\mathrm{k}}\right)_{2}=0$ for every $\mathrm{k} \geqslant \mathrm{N}+1$, so that the estimate

$$
\left\|\exp [\tau A] \mathrm{p}^{*}-\left(\mathrm{p}^{*}, \hat{\mathrm{q}}_{1}\right)_{2} \hat{\mathrm{p}}_{1}\right\|_{2} \leqslant c_{\mathrm{N}} \exp \left[-\tau\left|\nu_{\mathrm{N}}\right|\right]\left\|\mathrm{p}^{*}\right\|_{2}
$$

indeed holds with an obvious choice for $c_{\mathrm{N}}$, which immediately leads to (80).

Remarks. (1) Since $\lim _{\mathrm{k} \rightarrow+\infty} \nu_{\mathrm{k}}=0$ as a consequence of the compactness of $A$ (see, e.g., Theorem 7.1 in Chapter VII of [1]), Statement (c) of the corollary is very different from the corresponding assertions which one might get in finite-dimensional situations, as there is no spectral gap between $\nu_{1}=0$ and the remaining eigenvalues. Thus, our conclusion is that there is a large supply of initial conditions to choose from for any $N \in \mathbb{N}^{+}, N \geqslant 2$, such that the corresponding solutions to (5) stabilize exponentially rapidly to $\gamma \mathbf{p}_{\beta}$, Gibbs for some $\gamma \in \mathbb{C}$. Indeed, it is useful to recall here that $\hat{p}_{1}$ as given by (19) with $k=1$ is

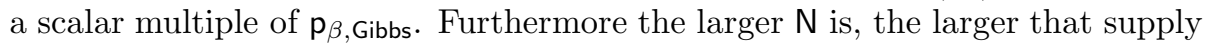
becomes but this is at the expense of having $\lim _{\mathrm{N} \rightarrow+\infty} \exp \left[-\tau\left|\nu_{\mathrm{N}}\right|\right]=1$. Therefore, there is a clear trade-off between the dimension of $\left(\mathrm{V}_{\mathrm{k}=\mathrm{N}+1}^{+\infty} E_{\nu_{\mathrm{k}}}\left(A^{*}\right)\right)^{\perp}$ and the rate of decay.

(2) The choice of the initial condition $\mathrm{p}^{*}$ in Statement (c) of Corollary 1 was motivated by the desire to generate the truncated expansion (81) in order to obtain the exponential rate of decay in (80). A natural question is therefore whether that truncation technique is really necessary to get at least some type of convergence toward a multiple of $\mathrm{p}_{\beta, \text { Gibbs }}$, in spite of the fact that $\nu_{1}=0$ is an accumulation point of $\sigma(A)$. So far we have been able to show that

$$
\lim _{\tau \rightarrow+\infty}\left\|\exp [\tau A] \mathrm{p}^{*}-\left(\mathrm{p}^{*}, \hat{\mathrm{q}}_{1}\right)_{2} \hat{\mathrm{p}}_{1}\right\|_{2}=0
$$

for every $\mathrm{p}^{*} \in l_{\mathbb{C}}^{1} \subset l_{\mathbb{C}}^{2}$, a very partial answer indeed as (82) does not provide rates of decay, nor does it say whether it holds for all $\mathrm{p}^{*} \in l_{\mathbb{C}}^{2}$ and not merely for all $\mathrm{p}^{*} \in l_{\mathbb{C}}^{1}$. Its proof is based on (51) written as

$$
\exp [\tau A] \mathrm{p}^{*}-\left(\mathrm{p}^{*}, \hat{\mathrm{q}}_{1}\right)_{2} \hat{\mathrm{p}}_{1}=\sum_{\mathrm{k}=2}^{+\infty}\left(\mathrm{p}^{*}, \hat{\mathrm{q}}_{\mathrm{k}}\right)_{2} \exp \left[\tau \nu_{\mathrm{k}}\right] \hat{\mathrm{p}}_{\mathrm{k}},
$$

and on a very careful estimate of the $l_{\mathbb{C}}^{2}$-norm of the right-hand side which rests on some of the inequalities proved in the core of this section. We omit the details.

(3) In many applications the real sequence $\left(\lambda_{\mathrm{m}}\right)_{\mathrm{m} \in \mathbb{N}^{+}}$used in the above considerations represents the pure point spectrum of some differential operator, 
typically a Hamiltonian operator in Quantum Mechanics. In this setting the coefficients $r_{\mathrm{m}, \mathrm{n}}$ given by (7) represent the transition rates between the corresponding eigenstates labeled $\mathrm{n}$ and $\mathrm{m}$, respectively, and equations of the form (3) may be used to describe the transient regime of a system that approaches thermodynamical equilibrium by using entropy production arguments, as was done at a formal level in Part D of Section II of 13 to which we refer the reader for details. Furthermore there are plenty of operators whose pure point spectrum satisfies (11), together with the conditions of all the propositions and theorems of this section, for instance the Hamiltonian operator describing the quantum harmonic oscillator. For an extension of the use of master equations as they relate to the investigation of physical or chemical systems in contact with reservoirs, we refer the reader again to [7] and [9] and also to Sections 10.4 and 10.5 in Chapter 10 of $[3$ where a functional-analytical treatment is carried out, and where many references to the physics litterature are given.

(4) Entropy production arguments may also be used to generalize the investigations carried out in [17 regarding a special class of stochastic processes, to which we can associate time-dependent entropy functionals of the form

$$
\mathrm{S}(\tau)=\sum_{\mathrm{m}=1}^{+\infty} p_{\mathrm{m}}(\tau) \ln \frac{1}{p_{\mathrm{m}}(\tau)}
$$

where $p_{\mathrm{m}}(\tau)$ is a solution to (3). The presentation of the related results is deferred to a separate publication.

We complete this article with the two appendices we alluded to in the introduction.

Appendix A. On the holomorphic continuation of the function given by (25).

The following result holds:

Proposition A.1. Let $\hat{f}: \mathbb{C} \backslash\left\{0,-b_{\mathrm{m}}, \mathrm{m} \in \mathbb{N}^{+}\right\} \mapsto \mathbb{C}$ be the function defined by

$$
\hat{f}(\nu):=\sum_{\mathrm{m}=1}^{+\infty} \frac{\exp \left[-\alpha \beta \lambda_{\mathrm{m}}\right]}{\nu+b_{\mathrm{m}}}
$$

where $b_{\mathrm{m}}$ is given by (18). Then $\hat{f}$ is holomorphic throughout its domain and we have

$$
\hat{f}^{\prime}(\nu)=-\sum_{\mathrm{m}=1}^{+\infty} \frac{\exp \left[-\alpha \beta \lambda_{\mathrm{m}}\right]}{\left(\nu+b_{\mathrm{m}}\right)^{2}} .
$$

Proof. The absolute convergence of (83) in every point of its domain is proved as for the function given by (25). Now let $\nu_{*} \in \mathbb{C} \backslash\left\{0,-b_{\mathrm{m}}, \mathrm{m} \in \mathbb{N}^{+}\right\}$ be arbitrary, and let

$$
\hat{f}_{\mathrm{N}}(\nu):=\sum_{\mathrm{m}=1}^{\mathrm{N}} \frac{\exp \left[-\alpha \beta \lambda_{\mathrm{m}}\right]}{\nu+b_{\mathrm{m}}}
$$


be the holomorphic partial sums of (83). In order to prove that $\hat{f}$ is holomorphic at $\nu_{*}$ it is sufficient to prove that $\hat{f}_{\mathrm{N}} \rightarrow \hat{f}$ uniformly on a compact disk of sufficiently small radius $R_{\nu_{*}}$ centered at $\nu_{*}$. To this end we consider

$$
\mathbb{D}_{R_{\nu_{*}}}\left(\nu_{*}\right)=\left\{\nu \in \mathbb{C}:\left|\nu-\nu_{*}\right| \leq R_{\nu_{*}}\right\}
$$

with $0<R_{\nu_{*}}<\left|\nu_{*}\right|$ and show that

$$
\sup _{\nu \in \mathbb{D}_{R_{\nu_{*}}}\left(\nu_{*}\right)}\left|\hat{f}(\nu)-\hat{f}_{\mathrm{N}}(\nu)\right| \leq \frac{2}{\left|\nu_{*}\right|-R_{\nu_{*}}} \sum_{\mathrm{m}=\mathrm{N}+1}^{+\infty} \exp \left[-\alpha \beta \lambda_{\mathrm{m}}\right]
$$

for $\mathrm{N}$ sufficiently large, which indeed implies the desired convergence by virtue of (11). Owing to the choice of $R_{\nu_{*}}$ we first have

$$
\begin{aligned}
& \left|\hat{f}(\nu)-\hat{f}_{\mathrm{N}}(\nu)\right| \\
\leq & \frac{1}{\left|\nu_{*}\right|-\left|\nu-\nu_{*}\right|} \sum_{\mathrm{m}=\mathrm{N}+1}^{+\infty} \frac{\exp \left[-\alpha \beta \lambda_{\mathrm{m}}\right]}{\left|1+\frac{b_{\mathrm{m}}}{\nu}\right|} \\
\leq & \frac{1}{\left|\nu_{*}\right|-R_{\nu_{*}}} \sum_{\mathrm{m}=\mathrm{N}+1}^{+\infty} \frac{\exp \left[-\alpha \beta \lambda_{\mathrm{m}}\right]}{\left|1+\frac{b_{\mathrm{m}}}{\nu}\right|} .
\end{aligned}
$$

Furthermore, since $b_{\mathrm{m}} \rightarrow 0$ as $\mathrm{m} \rightarrow+\infty$ there exists $\mathrm{N}_{\nu_{*}} \in \mathbb{N}^{+}$such that

$$
b_{\mathrm{m}} \leq \frac{\left|\nu_{*}\right|-R_{\nu_{*}}}{2}
$$

for every $\mathrm{m} \geq \mathrm{N}_{\nu_{*}}$, which implies the estimate

$$
\left|1+\frac{b_{\mathrm{m}}}{\nu}\right| \geq 1-\frac{b_{\mathrm{m}}}{\left|\nu_{*}\right|-R_{\nu_{*}}} \geq \frac{1}{2}
$$

uniformly in $\nu$. Using (87) in (86) with $\mathrm{N} \geq \mathrm{N}_{\nu_{*}}$ then leads to (85), so that $\hat{f}$ is holomorphic at $\nu_{*}$ and hence in $\mathbb{C} \backslash\left\{0,-b_{\mathrm{m}}, \mathrm{m} \in \mathbb{N}^{+}\right\}$since $\nu_{*}$ was arbitrary.

Similar estimates allow one to prove that $\hat{f}_{\mathrm{N}}^{\prime} \rightarrow \hat{f}^{\prime}$ uniformly on disks of sufficiently small radii where

$$
\hat{f}_{\mathrm{N}}^{\prime}(\nu)=-\sum_{\mathrm{m}=1}^{\mathrm{N}} \frac{\exp \left[-\alpha \beta \lambda_{\mathrm{m}}\right]}{\left(\nu+b_{\mathrm{m}}\right)^{2}},
$$

eventually establishing (84) (see, e.g., Section 3.5 in Chapter 3 of [6]).

Since $f$ given by (25) is the restriction of $\hat{f}$ to $(-\infty, 0) \backslash\left\{-b_{\mathrm{m}}, \mathrm{m} \in \mathbb{N}^{+}\right\}$, the preceding result justifies a posteriori the few properties of that function we used in the proof of Statement (c) of Theorem 1 regarding the localization of the eigenvalues $\nu_{\mathrm{k}}$. 
Appendix B. A simple characterization of the eigenvectors of $A^{*}$

In this short appendix we wish to characterize in a geometric way the sequence $\left(\hat{\mathrm{q}}_{\mathrm{k}}\right)_{\mathrm{k} \in \mathbb{N}^{+}}$biorthogonal to $\left(\hat{\mathrm{p}}_{\mathrm{k}}\right)_{\mathrm{k} \in \mathbb{N}^{+}}$constructed in the proof of Proposition 4. Let us consider the direct sum decomposition

$$
l_{\mathbb{C}}^{2}=\bigvee_{\mathrm{k}=1, \mathrm{k} \neq \mathrm{j}}^{+\infty} E_{\nu_{\mathrm{k}}}(A) \oplus\left(\bigvee_{\mathrm{k}=1, \mathrm{k} \neq \mathrm{j}}^{+\infty} E_{\nu_{\mathrm{k}}}(A)\right)^{\perp}
$$

for every $\mathrm{j} \in \mathbb{N}^{+}$, where the first space on the right-hand side stands for the closed linear hull of $\cup_{\mathrm{k}=1, \mathrm{k} \neq \mathrm{j}}^{+\infty} E_{\nu_{\mathrm{k}}}(A)$. We then consider the orthogonal projection

$$
Q_{\mathrm{j}}: l_{\mathbb{C}}^{2} \mapsto\left(\bigvee_{\mathrm{k}=1, \mathrm{k} \neq \mathrm{j}}^{+\infty} E_{\nu_{\mathrm{k}}}(A)\right)^{\perp}
$$

and remark that $Q_{\mathrm{j}} \hat{\mathrm{p}}_{\mathrm{j}} \neq 0$ for every $\mathrm{j}$. Indeed, $Q_{\mathrm{j}^{*}} \hat{\mathrm{p}}_{\mathrm{j}^{*}}=0$ for at least one $\mathrm{j}^{*}$ would mean that

$$
\hat{\mathrm{p}}_{\mathrm{j}^{*}} \in \operatorname{ker} Q_{\mathrm{j}^{*}}=\bigvee_{\mathrm{k}=1, \mathrm{k} \neq \mathrm{j}^{*}}^{+\infty} E_{\nu_{\mathrm{k}}}(A),
$$

which in turn would entail the relation

$$
\operatorname{span} \cup_{\mathrm{k}=1}^{+\infty} E_{\nu_{\mathrm{k}}}(A) \subseteq \bigvee_{\mathrm{k}=1, \mathrm{k} \neq \mathrm{j}^{*}}^{+\infty} E_{\nu_{\mathrm{k}}}(A) .
$$

But by taking the closure of (88) in $l_{\mathbb{C}}^{2}$ and by using (45) we would then obtain

$$
l_{\mathbb{C}}^{2} \subseteq \bigvee_{\mathrm{k}=1, \mathrm{k} \neq \mathrm{j}^{*}}^{+\infty} E_{\nu_{\mathrm{k}}}(A) \subset l_{\mathbb{C}}^{2},
$$

a contradiction. We may therefore consider the sequence $\left(\hat{\mathrm{t}}_{\mathrm{j}}\right)_{\mathrm{j} \in \mathbb{N}^{+}}$given by

$$
\hat{\mathrm{t}}_{\mathrm{j}}:=\left\|Q_{\mathrm{j}} \hat{\mathrm{p}}_{\mathrm{j}}\right\|_{2}^{-2} Q_{\mathrm{j}} \hat{\mathrm{p}}_{\mathrm{j}},
$$

from which we easily see that

$$
\left(\hat{\mathrm{p}}_{\mathrm{j}}, \hat{\mathrm{t}}_{\mathrm{k}}\right)_{2}=\delta_{\mathrm{j}, \mathrm{k}}
$$

for all $j, k \in \mathbb{N}^{+}$. Consequently, the sequence $\left(\hat{t}_{j}\right)_{j \in \mathbb{N}^{+}}$is biorthogonal to $\left(\hat{p}_{k}\right)_{k \in \mathbb{N}^{+}}$ and the preceding relation together with (54) immediately imply that

$$
\hat{\mathrm{t}}_{\mathrm{j}}=\hat{\mathrm{q}}_{\mathrm{j}}
$$

for every $\mathrm{j} \in \mathbb{N}^{+}$since the $\hat{p}_{\mathrm{j}}$ form a complete system in $l_{\mathbb{C}}^{2}$. Thus the sequence $\left(\hat{\mathrm{q}}_{\mathrm{k}}\right)_{\mathrm{k} \in \mathbb{N}^{+}}$of Proposition 4 is the unique sequence biorthogonal to $\left(\hat{\mathrm{p}}_{\mathrm{k}}\right)_{\mathrm{k} \in \mathbb{N}^{+}}$, and (89) with (90) provide a simple geometric characterization of the eigenvectors of $A^{*}$.

Acknowledgements. The second author would like to thank the Fundação para a Ciência e a Tecnologia (FCT) of the Portuguese Government for its financial support under grant PDTC/MAT-STA/0975/2014. 


\section{References}

[1] Conway, J. B., A Course in Functional Analysis, Graduate Texts in Mathematics 96, Springer Verlag, New York (1990).

[2] Daleckil, Ju. L., Krein, M. G., Stability of Solutions of Differential Equations in Banach Space, Translations of Mathematical Monographs 43, American Mathematical Society, Providence (1974).

[3] Davies, E. B., Quantum Theory of Open Systems, Academic Press, New York (1976).

[4] Gelfand, I. M., Vilenkin, N.Ya., Generalized Functions, Vol. 4: Applications of Harmonic Analysis, Academic Press, New York (1964).

[5] Gohberg, I. C., Krein, M. G., Introduction to the Theory of Linear Nonselfadjoint Operators in Hilbert Space, Translations of Mathematical Monographs 18, American Mathematical Society, Providence (1969).

[6] Greene, R. E., Krantz, S. G., Function Theory of One Complex Variable, Graduate Studies in Mathematics 40, American Mathematical Society, Providence (2006).

[7] HaAke, F., Statistical treatment of open systems by generalized master equations, in: Springer Tracts in Modern Physics 66, Springer, New York (1973).

[8] KeLdy $\breve{S}$, M. V., On the characteristic values and characteristic functions of certain classes of non self-adjoint equations, Doklady Akad. Nauk SSSR 77, 11-14 (1951).

[9] Mozgunov, E., Lidar, D., Completely positive master equation for arbitrary driving and small level spacing, Quantum 4, 227-289 (2020).

[10] Paley, R. E. A. C., Wiener, N., Fourier Transforms in the Complex Plane, American Mathematical Society Colloquium Publications XIX, American Mathematical Society, New York, (1934).

[11] Riesz, F., Nagy, B. SZ., Functional Analysis, Dover Books in Mathematics, Dover, (1990).

[12] Schnakenberg, J., Network theory of microscopic and macroscopic behavior of master equation systems, Reviews of Modern Physics 48, 571-585 (1976).

[13] Tomé, T., DE OliveIra, M. J., Stochastic approach to equilibrium and nonequilibrium thermodynamics, Physical Review E 91, 042140 (2015).

[14] Tomé, T., DE Oliveira, M. J., Stochastic thermodynamics and entropy production of chemical reaction systems, Journal of Chemical Physics 148, 224104 (2018). 
[15] van Kampen, N. G., Stochastic Processes in Physics and Chemistry, Elsevier Science Publishers B. V., Amsterdam (1981).

[16] Vinberg, E. B., A Course in Algebra, Graduate Studies in Mathematics 56, American Mathematical Society, Providence, Rhode Island (2003).

[17] Vuillermot, P.-A., On Bernstein processes of maximal entropy, Stochastic Analysis and Applications 38, 886-908 (2020). 